\title{
Preference Reversals and Effects of d-Amphetamine on Within- Session Delay Discounting in Rats
}

Christopher A. Krebs

West Virginia University

Follow this and additional works at: https://researchrepository.wvu.edu/etd

\section{Recommended Citation}

Krebs, Christopher A., "Preference Reversals and Effects of d-Amphetamine on Within-Session Delay Discounting in Rats" (2011). Graduate Theses, Dissertations, and Problem Reports. 3316.

https://researchrepository.wvu.edu/etd/3316

This Thesis is protected by copyright and/or related rights. It has been brought to you by the The Research Repository @ WVU with permission from the rights-holder(s). You are free to use this Thesis in any way that is permitted by the copyright and related rights legislation that applies to your use. For other uses you must obtain permission from the rights-holder(s) directly, unless additional rights are indicated by a Creative Commons license in the record and/ or on the work itself. This Thesis has been accepted for inclusion in WVU Graduate Theses, Dissertations, and Problem Reports collection by an authorized administrator of The Research Repository @ WVU. For more information, please contact researchrepository@mail.wvu.edu. 


\title{
Preference Reversals and Effects of $d$-Amphetamine on Within-Session Delay Discounting in Rats
}

\author{
Christopher A. Krebs
}

Thesis submitted to the Eberly College of Arts and Sciences at West Virginia University in partial fulfillment of the requirements for the degree of

Master of Science

in

Psychology

\author{
Karen G. Anderson, Ph.D., Chair \\ Michael Perone, Ph.D. \\ Kevin Larkin, Ph.D. \\ Department of Psychology
}

Morgantown, West Virginia

2011

Keywords: choice, $d$-amphetamine, delay discounting, preference reversals, rats 


\author{
ABSTRACT \\ Preference Reversals and Effects of d-Amphetamine on \\ Within-Session Delay Discounting in Rats
}

Christopher A. Krebs

Impulsive choice is correlated with behavioral problems such as attention-deficit/hyperactivitydisorder and can be assessed using delay-discounting procedures in which subjects choose between a smaller, more immediate reinforcer (impulsive choice) and a larger, more delayed reinforcer (self-controlled choice). A within-session delay-discounting procedure in which choice was between one food pellet delivered immediately and three food pellets delivered after increasing delays was used to examine effects of adding or subtracting delays common to both reinforcers on impulsive choice in male Sprague-Dawley rats $(\mathrm{N}=8)$. For all subjects, delay discounting was observed regardless of whether delays common to both reinforcers were added or subtracted. Using area under the curve (AUC) to quantify impulsive choice, adding delays common to both reinforcers decreased impulsive choice whereas subtracting delays common to both reinforcers increased impulsive choice. Prior to $d$-amphetamine administration, subjects were rank ordered into High-impulsive or Low-impulsive groups using AUC at the final delay condition. Subjects in the High-impulsive group made more impulsive choices than subjects in the Low-impulsive group and effects of $d$-amphetamine (0.03, 0.1, 0.18, 0.3, 0.56, 1.0, 1.8 $\mathrm{mg} / \mathrm{kg}$ ) generally depended on these differences. Select doses of $d$-amphetamine decreased impulsive choice for subjects in the High-impulsive group but not for subjects in the Lowimpulsive group. These results show that levels of impulsive choice can be altered by changing the delay common to both reinforcers and suggest that effects of $d$-amphetamine on impulsive choice are determined, in part, by baseline levels of impulsive choice. 


\section{Acknowledgements}

I would like to thank Karen Anderson, Mike Perone, and Kevin Larkin for serving as members of my thesis committee and for their valuable comments in preparing this manuscript. I am particularly thankful to Karen for serving as my committee chair and advisor over the past three years. 
Table of Contents

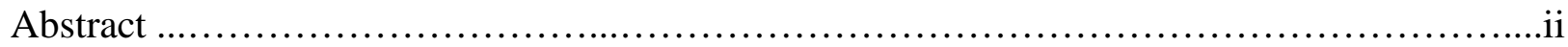

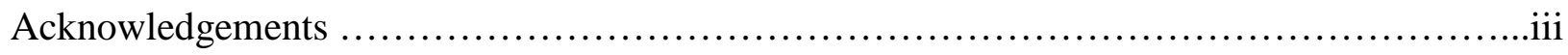

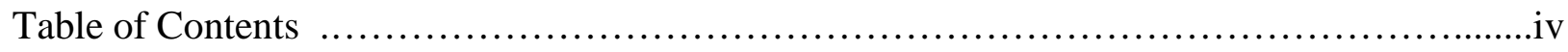

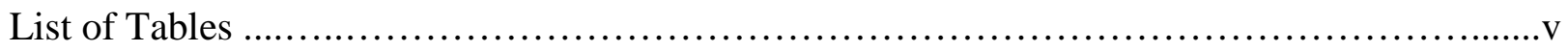

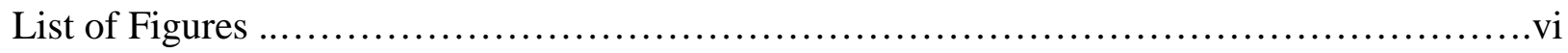

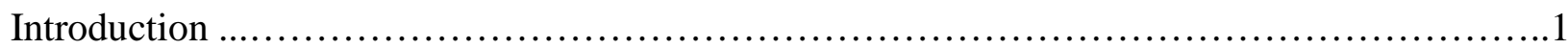

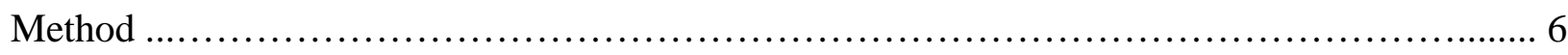

Subjects................................................................. 6

Apparatus...................................................................... 6

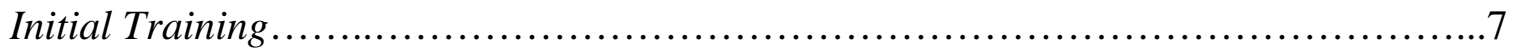

Delay-Discounting Procedure................................................

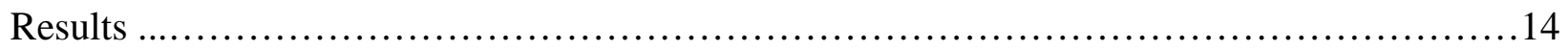

Delay Conditions ............................................................14

Acute d-Amphetamine Phase................................................23

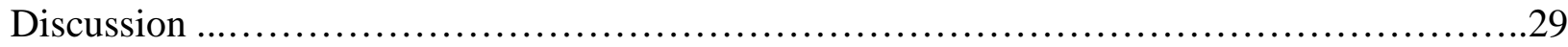

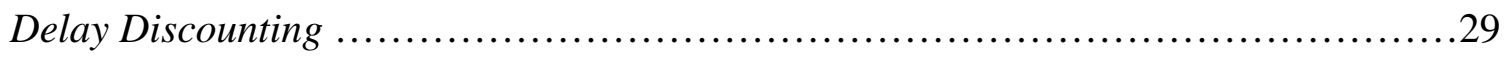

Effects of $d$-Amphetamine .................................................. 31

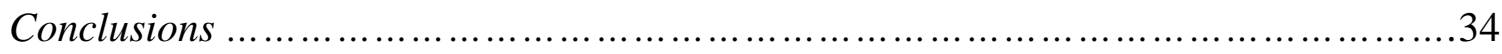

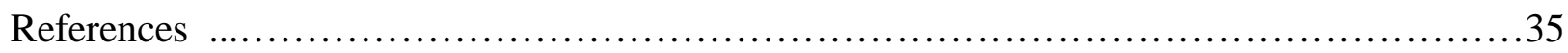




\section{List of Tables}

Table 1. The number of sessions conducted until stability and the number of probe sessions conducted during each delay condition for subjects in the delay-added (A) and delay-subtracted (S) groups. Means and standard error of the means are shown for the delay-added and delaysubtracted groups. Asterisks represent group differences that were significant at $p<.05 \ldots . . . .15$

Table 2. Percent larger-reinforcer choice in each block of trials and area under the curve (AUC) averaged across the last five sessions during each delay condition with standard error of the means in parentheses for subjects in the delay-added (A) or delay-subtracted (S) groups ......20

Table 3. Percent larger-reinforcer choice and area under the curve (AUC) averaged across control sessions and each dose of $d$-amphetamine administered for individual subjects in the High-impulsive group with standard error of the means in parentheses. The number of control sessions and administrations of each dose of $d$-amphetamine are in parentheses. The largerreinforcer delay series (s) in effect during $d$-amphetamine administration is also shown. Asterisks represent differences in AUC compared to saline that were significant at $p<.05$ and crosses represent doses of $d$-amphetamine that decreased percent larger-reinforcer choice in the first block of free-choice trials below $80 \%$

Table 4. Percent larger-reinforcer choice and area under the curve (AUC) averaged across control sessions and each dose of $d$-amphetamine administered for individual subjects in the Low-impulsive group. The number of control sessions and administrations of each dose of $d$ amphetamine are in parentheses. The larger-reinforcer delay series (s) in effect during $d$ amphetamine administration is also shown. The asterisk represent differences in AUC compared to saline that were significant at $p<.05$ and crosses represent doses of $d$-amphetamine that decreased percent larger-reinforcer choice in the first block of free-choice trials below $80 \%$...25 


\section{List of Figures}

Figure 1. Percent larger-reinforcer choice averaged across subjects in the delay-added and delay-subtracted groups for the last five sessions (filled circles) and for the last probe session conducted (unfilled circle) during the 0-s (top-left panel, a), 2-s (top-right panel, b), 4-s (bottomleft panel, c), and 8-s (bottom-right panel, d) delay conditions as a function of block of trials. Error bars are \pm 1 SEM. The delay condition and number of subjects exposed to that delay condition are shown above the $x$-axis in each panel. Only CK-1 and CK-7 were exposed to the 8-s delay condition

Figure 2. Area under the curve (AUC) averaged across subjects in the delay-added and delaysubtracted groups for the last five sessions during each delay condition. Error bars are \pm 1 SEM. Individual subject data are represented by the respective subject number (i.e., CK-1 = 1). Only CK-1 and CK-7 were exposed to the 8-s delay condition. Asterisks represent differences in AUC

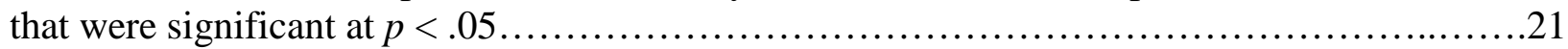

Figure 3. Area under the curve (AUC) for subjects in the High-impulsive and Low-impulsive groups during the final delay condition. Error bars are \pm 1 SEM. Individual subject data are represented by the respective subject numbers (i.e., CK-1 = 1). Data for CK-2 and CK-7 (Highimpulsive group) overlap and data for CK-6 (Low-impulsive group) were obtained before reexposure to delay conditions. The asterisk represents difference in AUC that was significant at $p$

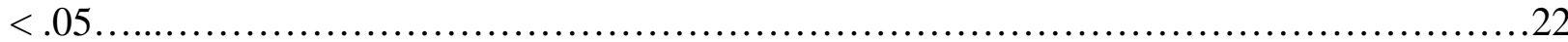

Figure 4. Percent of saline AUC (dashed horizontal lines in each panel) for subjects in the Highimpulsive group for control sessions (C) and each dose of $d$-amphetamine administered. Subjects are identified above the $x$-axis in each panel. The $y$-axis is scaled differently for CK-4. Asterisks represent doses of $d$-amphetamine that changed AUC compared to saline that were significant at $p<.05$ and crosses represent doses that decreased percent larger-reinforcer choice during the first block of free-choice trials below $80 \%$

Figure 5. Percent of saline AUC (dashed horizontal lines in each panel) for subjects in the Lowimpulsive group for control sessions (C) and each dose of $d$-amphetamine administered. Subjects are identified above the $x$-axis in each panel. The asterisk represent a dose of $d$ amphetamine that changed AUC compared to saline that was significant at $p<.05$ and crosses represent doses that decreased percent larger-reinforcer choice during the first block of freechoice trials below $80 \%$ 
Introduction

Impulsive choice is correlated with maladaptive behaviors such as attentiondeficit/hyperactivity disorder (ADHD) (Barkley \& Biederman, 1997), substance abuse (Bickel, Odum, \& Madden, 1999; Kirby \& Petry, 2004; Vuchinich \& Simpson, 1998), gambling (Alessi \& Petry, 2003), and aggression (Cherek \& Lane, 1999; Ramirez \& Andreu, 2006). Impulsive choice has been operationally defined as choice for a smaller, more immediate reinforcer over a larger, more delayed reinforcer and the opposite, a self-controlled choice, as choice for a larger, more delayed reinforcer over a smaller, more immediate reinforcer (Ainslie, 1975; Logue, 1988; Mazur, 1987; Rachlin \& Green, 1972). In the laboratory, impulsive choice in human and nonhuman subjects can be studied using delay-discounting procedures. In these procedures, subjects are typically presented with a series of choices between two reinforcers of different magnitudes presented at varying delays. The delay to the smaller reinforcer usually remains constant while the delay to the larger reinforcer is adjusted within or between experimental sessions. When the delay to the larger reinforcer is short or absent, choice is typically for the larger reinforcer. As the delay to the larger reinforcer increases, however, choice for the larger reinforcer decreases and the value of the larger reinforcer is said to be discounted (Mazur). Thus, two known determinants of impulsive choice are reinforcer amount and delay.

A discrete-trials choice procedure based on procedures developed by Evenden and Ryan (1996; 1999) has been used to generate delay-discounting functions within a single experimental session (e.g., Anderson \& Diller, 2010; Anderson \& Woolverton, 2005; Slezak \& Anderson, 2009). This within-session delay-discounting procedure consists of five, eight-trial blocks in which the delay to the larger reinforcer (three food pellets) is increased across blocks of trials (e.g., 0, 10, 20, 40, $60 \mathrm{~s}$ ) while the delay to the smaller reinforcer (one food pellet) remains 
constant (e.g., 0 s). The first two trials in each block are forced-exposure trials that allow contact with the contingencies programmed for each block of trials. During forced-exposure trials, one alternative is presented during the first trial and the other alternative is presented during the second trial. The remaining six trials are free-choice trials in which the alternatives presented during forced-exposure trials are presented simultaneously. Choice for the larger reinforcer typically decreases as the delay to the larger reinforcer is increased and plotting percent largerreinforcer choice as a function of the delay to the larger reinforcer generates delay-discounting functions after a single session that can serve as baselines from which effects of other variables such as drugs can be evaluated (Anderson \& Diller; Anderson \& Woolverton; Cardinal, Robbins, \& Everitt, 2000; Diller, Saunders, \& Anderson, 2008; Huskinson, Krebs, \& Anderson, 2011; Slezak \& Anderson).

\section{Stimulant Drugs}

Drugs classified as stimulants (e.g., methylphenidate, amphetamine) have been shown to decrease impulsive choice in clinical populations (Barkley, 1977; Kollins, MacDonald \& Rush, 2001; Pietras, Cherek, Lane, Tcheremissine, Steinberg, 2003). In the laboratory, however, effects of stimulant drugs on impulsive choice (measured using delay-discounting procedures) have been mixed. For example, acute administration of stimulant drugs has been shown to decrease impulsive choice (Cardinal, Robbins, \& Everitt, 2000; Diller, Saunders, \& Anderson, 2008; Pitts \& Febbo, 2004; Pitts \& McKenney, 2005; Richards, Sabol, \& de Wit, 1999; Wade, de Wit, \& Richards, 2000; Winstanley, Dallery, Theobald, \& Robins, 2003) or increase impulsive choice (Cardinal, Robbins, \& Everitt, 2000; Charrier \& Theibot, 1996; Evenden \& Ryan, 1996; Slezak \& Anderson, 2009). The factors responsible for the discrepant effects of stimulant drugs on impulsive choice have not been conclusively identified, but factors such as the specific drugs 
used, stimulus conditions during delays, and differences in baseline levels of impulsive choice may be relevant.

Baseline levels of impulsive choice have been shown to depend on the presence or absence of stimuli in the rearing environment. Perry, Stairs, and Bardo (2008), for example, reported that rats reared in enriched environments (with conspecifics and plastic objects) made fewer impulsive choices during baseline than rats reared in isolated environments (without conspecifics and plastic objects). Moreover, effects of stimulant drugs on impulsive choice depended on these baseline differences in impulsive choice. Acute administration of $d$-amphetamine and methylphenidate decreased impulsive choice in rats that made more impulsive choices during baseline (those reared in isolated environments). For rats that made fewer impulsive choices during baseline (those reared in enriched environments), however, $d$-amphetamine increased impulsive choice and methylphenidate had no effect.

Baseline-dependent effects of stimulant drugs on impulsive choice have been reported in other studies using delay-discounting procedures (Barbelivien, Billy, Lazarus, Kelche, \& Majchrzak, 2007; Hand, Fox, \& Reilly, 2009) and in studies using different measures of impulsive choice (Eagle \& Robbins, 2003; Eagle, Tufft, Goodchild, \& Robbins, 2007; Feola, de Wit, \& Richards, 2000). Additional research is needed to identify the specific environmental variables responsible for generating different levels of impulsive choice and to determine how those variables influence effects that stimulant drugs have on impulsive choice.

\section{Preference Reversals}

In delay-discounting contexts, choice can be thought of as being determined by the subjective value of the potential reinforcers at the moment the choice is made (Green \& Myerson, 2004). Thus, if a smaller, more immediate reinforcer is chosen over a larger, more 
delayed reinforcer, it can be said that the subjective value of the smaller, more immediately available reinforcer was greater than the subjective value of the larger, more delayed reinforcer at the moment the choice was made (Mazur, 1987). One way to alter choice for a smaller, more immediate reinforcer or a larger, more delayed reinforcer is to adjust the delay common to both reinforcers. For example, if choice is initially for a smaller, more immediate reinforcer over a larger, more delayed reinforcer, adding a delay common to both reinforcers has been shown to increase choice for the larger, more delayed reinforcer (Ainslie \& Herrnstein, 1981; Green \& Myerson). The change in choice, from an impulsive choice to a self-controlled choice, is referred to as a preference reversal and has been shown to occur in both human (Green, Fristoe, \& Myerson, 2004; Green, Myerson, \& Macaux, 2005) and non-human (Ainslie \& Herrnstein; Deluty, 1978; Green, Fischer, Perlow, \& Sherman, 1981; Green \& Estle) subjects.

Ainslie and Herrnstein (1981), for example, generated preference reversals in pigeons with food reinforcement using a discrete-trials choice procedure in which choice was always between a smaller, more immediate reinforcer and a larger, more delayed reinforcer. For the entire experiment, the larger reinforcer was delayed $4 \mathrm{~s}$ longer than the smaller reinforcer and across conditions the delay to the smaller reinforcer was systematically increased or decreased. When the delay to the smaller reinforcer was relatively short, choice for the smaller reinforcer was almost exclusive; however, as the delay to the smaller reinforcer increased, choice for the larger reinforcer increased for all pigeons.

Preference reversals have also been shown to occur in rats using qualitatively different reinforcers such as food and water (Green \& Estle, 2003) and shock delivery (Deluty, 1978). Green and Estle, for example, generated preference reversals for both food and water reinforcers within subjects using a discrete-trials choice procedure. Choice was always between a smaller, 
more immediate reinforcer and a larger, more delayed reinforcer and the type of reinforcer used was counterbalanced across subjects and between experiments. For both food and water reinforcers, the delay to the larger reinforcer was initially adjusted for each subject to ensure that the majority of choices (i.e., at least 70\%) were for the smaller reinforcer. Across conditions, choice for the larger reinforcer increased as the delay common to both reinforcers increased.

The preference reversal literature indicates that increasing the delay common to both reinforcers reliably increases choice for larger, more delayed reinforces (i.e., decreases impulsive choice) and that decreasing the delay common to both reinforcers increases choice for smaller, more immediate reinforcers (i.e., increases impulsive choice). These findings suggest that the delay common to both reinforcers is an important determinant of impulsive choice and that altering the delay common to both reinforcers may be one way to generate different levels of impulsive choice. Furthermore, if different levels of impulsive choice can be generated by altering the delay common to both reinforcers, effects of stimulant drugs such as $d$-amphetamine on impulsive choice could be evaluated across subjects with different levels of impulsive choice.

\section{Statement of the Problem}

In the laboratory, effects of stimulant drugs on impulsive choice are mixed and these discrepancies may be due to different levels of impulsive choice (e.g., Perry, Stairs, \& Bardo, 2008). The purpose of the present experiment was to determine if individual levels of impulsive choice could be altered by increasing or decreasing the delay common to both reinforcers and if so, if effects of $d$-amphetamine depended on the level of impulsive choice. To this end, delays common to both reinforcers were added to (delay-added group) or subtracted from (delaysubtracted group) the delays in effect using a within-session delay-discounting procedure. Adding delays common to both reinforcers was expected to decrease impulsive choice whereas 
subtracting delays common to both reinforcers was expected to increase impulsive choice. Moreover, $d$-amphetamine was expected to increase impulsive choice for subjects with low levels of impulsive choice (delay-added group) and decrease impulsive choice for subjects with high levels of impulsive choice (delay-subtracted group).

\section{Method}

\section{Subjects}

Eight experimentally naïve male Sprague-Dawley rats (Harlan Sprague-Dawley, Inc. Indianapolis, IN) served as subjects (CK-1 - CK-8). Subjects were individually housed under a 12-hr reversed light-dark cycle in a colony room where temperature and humidity were maintained at constant levels. Experimental sessions were conducted during the dark phase at approximately the same time each day. In home cages, water was continuously available and subjects received $15 \mathrm{~g}$ of rat chow approximately $30 \mathrm{~min}$ after experimental sessions. This resulted in about $22 \mathrm{hrs}$ of food restriction prior to the start of experimental sessions. These procedures were conducted in accordance with the Animal Care and Use Committee of West Virginia University.

\section{Apparatus}

Experimental sessions were conducted in eight identical rat operant-conditioning chambers, each enclosed in a sound-attenuating cubicle with a ventilation fan (Med Associates, VT). Each chamber contained a work area of $30.5 \mathrm{~cm}$ by $24.5 \mathrm{~cm}$ by $21.0 \mathrm{~cm}$, a grid floor, and a 45-mg pellet dispenser with a pellet receptacle centered between two retractable response levers that were $11.5 \mathrm{~cm}$ apart from each other. Levers were $4.8 \mathrm{~cm}$ wide, protruded $1.9 \mathrm{~cm}$ into the chamber, elevated $8 \mathrm{~cm}$ from the grid floor, and required a press of $0.25 \mathrm{~N}$ for a response to be recorded. Two 28-V stimulus lights, $2.5 \mathrm{~cm}$ in diameter, were mounted approximately $7 \mathrm{~cm}$ 
above each lever. In addition, each chamber contained a 28-V houselight on the wall opposite the pellet dispenser. Experimental sessions were programmed and data collected using MedPC software (Med Associates, VT) interfaced to a personal computer.

\section{Initial Training}

To establish lever pressing, all subjects were exposed to a conjoint fixed-ratio (FR) 1 variable-time (VT) 60-s schedule of reinforcement. Sessions began with illumination of the houselight and extension of both levers. A lever press on either lever resulted in the delivery of one food pellet and, independent of responding, one food pellet was delivered, on average, every 60 s. Delivery of a food pellet was accompanied by a houselight flash of $0.5 \mathrm{~s}$. Sessions ended after 40 food pellets were delivered.

After lever-press acquisition, all subjects were exposed to an alternating FR 1 schedule of reinforcement. At the start of these sessions, one lever (randomly determined) extended into the chamber and the cue light above it illuminated. A lever press on this lever resulted in the delivery of one food pellet. After five consecutive lever presses on this lever, it retracted and the cue light above it extinguished. The other lever then extended into the chamber and the cue light above it illuminated. After five consecutive lever presses on this lever, it retracted and the cue light above it extinguished. This alternating FR 1 schedule occurred within each session until 40 food pellets were delivered. Subjects were exposed to this procedure for four or five sessions.

\section{Delay-Discounting Procedure}

General Procedure. After initial training, subjects were exposed to a within-session delay-discounting procedure. Sessions began with a 10-min blackout followed by five, eighttrial blocks. Each block consisted of two forced-exposure trials and then six free-choice trials. At the start of the first forced-exposure trial, the houselight illuminated, one lever (randomly 
determined) extended into the chamber, and the cue light above it illuminated. A lever press on this lever retracted it, extinguished the cue light above it, delivered the outcome correlated with that lever, and then extinguished the houselight. During the second forced-exposure trial, the houselight illuminated, the other lever extended into the chamber, and the cue light above it illuminated. A lever press on this lever retracted it, extinguished the cue light above it, delivered the outcome correlated with that lever, and then extinguished the houselight. Forced-exposure trials preceded free-choice trials to allow subjects to contact the contingencies programmed for each lever before being allowed to choose between them. During free-choice trials, both levers extended into the chamber and their respective cue lights, as well as the houselight, were illuminated. A lever press on either lever retracted both levers, extinguished both cue lights, delivered the outcome correlated with the just pressed lever, and then extinguished the houselight.

Sessions were conducted seven days per week and ended after 40 trials (10 forcedexposure and 30 free-choice trials). Because trials started every $100 \mathrm{~s}$, varied inter-trial intervals (ITIs) (i.e., blackout) occurred across blocks of trials. If a lever press did not occur within $30 \mathrm{~s}$ of trial onset, that trial was recorded as an omission, the lever(s) retracted, the cue light(s) and the houselight extinguished, and a 70-s ITI began; if more than six omissions occurred during a session, data from that session were not used. For the entire experiment, a lever press on one lever resulted in delivery of a smaller reinforcer (one food pellet) whereas a lever press on the other lever resulted in delivery of a larger reinforcer (three food pellets). These outcomes associated were counterbalanced across subjects in each group (see below for one exception).

Control by Reinforcer Amount. Control by reinforcer amount was assessed for all subjects after they were randomly assigned to a delay-added $(n=4)$ or delay-subtracted $(n=4)$ 
group. For subjects in the delay-added group, the delay to both the smaller and larger reinforcers was $0 \mathrm{~s}$ across all blocks of trials and a lever press during either type of trial resulted in the delivery of the smaller or larger reinforcer after $0 \mathrm{~s}$. Control by reinforcer amount was assessed the same way for subjects in the delay-subtracted group except that the delay to both the smaller and larger reinforcers was $4 \mathrm{~s}$ across all blocks of trials. These sessions remained in effect until percent larger-reinforcer choice across each block of free-choice trials was at least 80\%. Delaydiscounting functions were then established for subjects in both groups by increasing the delay to the larger reinforcer across blocks of trials.

Delay-Added Group. Subjects in the delay-added group were first exposed to a 0-s delay condition during which the delay to the smaller reinforcer was always $0 \mathrm{~s}$ and the delay to the larger reinforcer increased from 0, 1, 2, 4, 6 s across blocks of trials. This larger-reinforcer delay series remained in effect for three sessions and until percent larger-reinforcer choice during the first block of free-choice trials was at least $80 \%$. Using these criteria, delays to the larger reinforcer were changed to $0,2,4,8,16$ s and then $0,5,10,20,40$ s. To obtain delaydiscounting functions without floor or ceiling effects, the larger-reinforcer delay series was adjusted for individual subjects if necessary. For example, if the majority of choices were for the larger reinforcer across blocks of trials (i.e., ceiling effects) when the larger-reinforcer delay series was $0,5,10,20,40 \mathrm{~s}$, the delay series would be changed to $0,10,20,40,60 \mathrm{~s}$. After a delay series was functionally determined, it remained in effect until choice was stable (see below).

Following stability during the 0-s delay condition, subjects were exposed to a 2-s and then a 4-s delay condition. During the 2-s delay condition, $2 \mathrm{~s}$ was added to the delays in effect during the 0 -s delay condition. For example, if the delay to the smaller reinforcer was $0 \mathrm{~s}$ and 
the delay to the larger reinforcer increased from $0,10,20,40,60$ s across blocks of trials during the 0-s delay condition, then during the 2-s delay condition the delay to the smaller reinforcer would be $2 \mathrm{~s}$ and the delay to the larger reinforcer would increase from 2, 12, 22, 42, 62 s across blocks of trials. Next, subjects were exposed to the 4-s delay condition during which $4 \mathrm{~s}$ was added to the delays in effect during the 0-s delay condition. For example, if the delay to the smaller reinforcer was $0 \mathrm{~s}$ and the delay to the larger-reinforcer increased from 0, 10, 20, 40, $60 \mathrm{~s}$ across blocks of trials during the 0-s delay condition, then during the 4-s delay condition the delay to the smaller reinforcer would be $4 \mathrm{~s}$ and the delay to the larger reinforcer would increase from 4, 14, 24, 44, 64 s across blocks of trials. Following stability during the 4-s delay condition, two subjects (CK-1 and CK-7) were exposed to an 8-s delay condition because the 2-s and 4-s delay conditions did not increase larger-reinforcer choice. The 8-s delay condition was similar to the 2-s and 4-s delay conditions except that $8 \mathrm{~s}$ was added to the delays in effect during the 0 -s delay condition. Acute effects of $d$-amphetamine were then determined for CK-1 and CK-7 following stability during the 8-s delay condition and for CK-3 and CK-5 following stability during the 4-s delay condition.

Delay-Subtracted Group. Subjects in the delay-subtracted group were first exposed to a 4-s delay condition during which the delay to the smaller reinforcer was $4 \mathrm{~s}$ and the delay to the larger reinforcer increased from 4, 5, 6, 8, 10 s across blocks of trials. This larger-reinforcer delay series remained in effect for three sessions and until percent larger-reinforcer choice during the first block of free-choice trials was at least $80 \%$. Using these criteria, delays to the larger reinforcer were changed to 4, 6, 8, 12, 20 s and then 4, 9, 14, 24, 44 s. To obtain delaydiscounting functions without floor or ceiling effects, the larger-reinforcer delay series was adjusted for individual subjects if necessary. For example, if the majority of choices were for the 
larger reinforcer across blocks of trials (i.e., ceiling effects) when the larger-reinforcer delay series was 4, 9, 14, 24, $44 \mathrm{~s}$, the delay series would be changed to 4, 14, 24, 44, $64 \mathrm{~s}$. After a delay series was functionally determined, it remained in effect until choice was stable.

Following stability during the 4-s delay condition, subjects were exposed to a 2-s and then a 0-s delay condition. During the 2-s delay condition, $2 \mathrm{~s}$ was subtracted from the delays in effect during the 4-s delay condition. For example, if the delay to the smaller reinforcer was $4 \mathrm{~s}$ and the delay to the larger reinforcer increased from 4, 14, 24, 44, 64 s across blocks of trials during the 4-s delay condition, then during the 2-s delay condition, the delay to the smaller reinforcer would be $2 \mathrm{~s}$ and the delay to the larger reinforcer would increase from 2, 12, 22, 42, $62 \mathrm{~s}$ across blocks of trials. Next, subjects were exposed to the 0-s delay condition during which $4 \mathrm{~s}$ was subtracted from the delays in effect during the 4-s delay condition. For example, if the delay to the smaller reinforcer was $4 \mathrm{~s}$ and the delay to the larger reinforcer increased from 4, 14, 24, 44, 64 s across blocks of trials during the 4-s delay condition, then during the 0-s delay condition, the delay to the smaller reinforcer would be $0 \mathrm{~s}$ and the delay to the larger reinforcer would increase from $0,10,20,40,60$ s across blocks of trials. Acute effects of $d$-amphetamine were then determined for CK-2, CK-4, CK-6, CK-8 following stability during the 0-s delay condition.

For CK-6, subtracting delays common to both reinforcers did not decrease largerreinforcer choice. Therefore, after effects of $d$-amphetamine were determined, the outcomes associated with the left and right levers were reversed and CK-6 was re-exposed to the 0-s, 2-s, and 4-s delay conditions as described for subjects in the delay-added group and larger-reinforcer choice increased as the delay common to both reinforcers increased. Because it is not clear why subtracting delays common to both reinforcers did not decrease larger-reinforcer choice for this 
subject, only data obtained during $d$-amphetamine administration (including the 0 -s delay condition prior to $d$-amphetamine administration) and during re-exposure to the delay conditions were included in the present report.

Probe Sessions. During each delay condition, probe sessions were conducted every Wednesday to assess control by reinforcer amount. Probe sessions were the same as regular sessions except that the delay to the smaller and larger reinforcers was the same across all blocks of trials. For example, during the 0 -s delay condition, the delay to both reinforcers during probe sessions was $0 \mathrm{~s}$ across all blocks of trials whereas during the 2-s delay condition it was $2 \mathrm{~s}$ across all blocks of trials. During the 4-s and 8-s delay conditions the delay to both reinforcers during probe sessions was $4 \mathrm{~s}$ or $8 \mathrm{~s}$ across all blocks of trials, respectively. Subsequent probe sessions were conducted until percent larger-reinforcer choice across all blocks of free-choice trials was at least $80 \%$.

Stability. Each delay condition was in effect for a minimum of twenty sessions. Stability was determined based on data obtained during the last five sessions and required: (a) that larger-reinforcer choice during the first block of free-choice trials be at least $80 \%$, (b) that variation in the total number of larger-reinforcer choices during free-choice trials be less than $20 \%$, (c) no increasing or decreasing trends in larger-reinforcer choice based on visual inspection of daily graphs, and (d) completion of the first weekly probe session.

Acute $d$-Amphetamine. Effects of $d$-amphetamine on impulsive choice were determined after choice was stable during the final delay condition for subjects in the delayadded and delay-subtracted groups. That is, effects of $d$-amphetamine were determined following stability during the 4-s delay condition for CK-3 and CK-5, the 8-s delay condition for CK-1 and CK-7, and the 0-s delay condition for CK-2, CK-4, CK-6, and CK-8. Saline or $d-$ 
amphetamine $(0.1,0.3,1.0,1.8 \mathrm{mg} / \mathrm{kg})$ were administered to all subjects. For some subjects, intermediate doses of $d$-amphetamine $(0.03,0.18,0.56 \mathrm{mg} / \mathrm{kg})$ were administered. Drug administration occurred on Tuesdays and Fridays if larger-reinforcer choice during the first freechoice block in the preceding session (control session) was at least 80\%. Effects of saline were determined at least once prior to $d$-amphetamine administration to ensure that there were no behavioral disruptions due to the injection procedure. The order of $d$-amphetamine administration was counterbalanced across subjects in each group. Saline administrations occurred between these sequences. All doses were administered at least twice, with additional administrations occurring when choice was variable at a particular dose.

Drugs. $d$-Amphetamine obtained from Sigma-Aldrich (St. Louis, MO, USA) was dissolved in $0.9 \%$ sodium chloride $(1.0 \mathrm{mg} / \mathrm{ml})$ and administered at a volume of $1.0 \mathrm{ml} / \mathrm{kg}$. Saline or $d$-amphetamine (0.03, 0.1, 0.18, 0.3, 0.56, 1.0, $1.8 \mathrm{mg} / \mathrm{kg}$ i.p.) was administered immediately prior to experimental sessions.

Data Analysis. Percent larger-reinforcer choice was the primary dependent measure. For each delay condition, discounting functions were plotted as percent larger-reinforcer choice averaged across the last five sessions across blocks of trials. Area under the curve (AUC) was used to quantify impulsive choice and it was calculated for the last five sessions for each delay condition, control session, and saline or drug administration. AUC was determined using the methods developed by Myerson, Green, and Warusawitharana (2001). Specifically, percent larger-reinforcer choice was plotted as a function of normalized delay value and vertical lines were drawn from each data point to the $x$-axis creating a series of trapezoids, the sum of which equaled AUC. Smaller AUC values are consistent with steeper discounting functions and higher 
levels of impulsive choice whereas larger AUC values are consistent with shallower discounting functions and lower levels of impulsive choice.

Repeated-measure ANOVAs with group (delay-added or delay-subtracted) as a betweensubjects factor and delay condition as a within-subject factor were conducted to assess differences in the number of sessions required to meet stability criteria, the number of probe sessions conducted, percent larger-reinforcer choice across delay blocks, and AUC for each delay condition. Planned comparisons were conducted using two-sample t tests because of the unequal number of subjects in each group. Two-sample t tests were also used to evaluate effects of $d$-amphetamine on AUC for individual subjects because not all subjects received the same number of or doses of $d$-amphetamine. For all analyses, results were considered statistically significant when $p<.05$.

\section{Results}

\section{Delay Conditions}

Sessions to Stability. Table 1 shows the number of sessions required to meet stability criteria and the number of probe sessions conducted during each delay condition for subjects in the delay-added and delay-subtracted groups. Although there were no significant interactions or main effects for either group with the number of sessions for delay condition or the number of probe sessions during each delay condition as the dependent measure, a two-sample t test indicated that significantly more sessions were required to meet stability criteria during the 0 -s delay condition for subjects in the delay-added group compared to subjects in the delaysubtracted group, $t(4)=2.92, p=.04$. This difference, however, was primarily due to two subjects in the delay-added group (CK-1 and CK-3) that required more than 50 sessions to meet stability during the 0-s delay condition. 
Table 1

The number of sessions conducted for each delay condition and the number of probe sessions conducted during each delay condition until choice was stable for subjects in the delay-added (A) and delay-subtracted (S) groups. Means and standard error of the means are shown for both groups. Asterisks represent differences that were significant at $p<.05$.

\begin{tabular}{|c|c|c|c|c|c|c|c|c|}
\hline \multirow[t]{2}{*}{ Subject } & \multicolumn{4}{|c|}{ Delay Condition } & \multicolumn{4}{|c|}{ Probe Sessions } \\
\hline & $0 \mathrm{~s}$ & $2 \mathrm{~s}$ & $4 \mathrm{~s}$ & $8 \mathrm{~s}$ & $0 \mathrm{~s}$ & $2 \mathrm{~s}$ & $4 \mathrm{~s}$ & $8 \mathrm{~s}$ \\
\hline CK-1 (A) & 51 & 43 & 27 & 24 & 25 & 27 & 16 & $2^{\mathrm{a}}$ \\
\hline CK-3 (A) & 59 & 40 & 29 & $-{ }^{b}$ & 38 & 13 & 6 & $-\mathrm{b}$ \\
\hline CK-5 (A) & 27 & 25 & 20 & $-{ }^{b}$ & 10 & 6 & 4 & $-{ }^{b}$ \\
\hline CK-6 (A) ${ }^{\mathrm{C}}$ & 26 & 22 & 23 & $-\mathrm{b}$ & 5 & 10 & 5 & $-\mathrm{b}$ \\
\hline CK-7 (A) & 35 & 52 & 23 & 44 & 17 & 27 & 11 & 23 \\
\hline $\begin{array}{l}\text { Delay- } \\
\text { Added } \\
M(S E M)\end{array}$ & $39.6(6.5)^{*}$ & $36.4(5.0)$ & $24.4(1.8)$ & $34.0(6.3)$ & $19.0(5.4)$ & $16.6(4.7)$ & $8.4(2.4)$ & $12.5(6.6)$ \\
\hline CK-2 (S) & 20 & 23 & 20 & $-b$ & 5 & 13 & 5 & $-b$ \\
\hline CK-4 (S) & 21 & 23 & 20 & $-{ }^{b}$ & 8 & 6 & 3 & $-\mathrm{b}$ \\
\hline CK-8 (S) & 20 & 36 & 36 & $-{ }_{-}^{b}$ & 4 & 6 & 13 & $-\mathrm{b}$ \\
\hline $\begin{array}{l}\text { Delay- } \\
\text { Subtracted } \\
M(\text { SEM })\end{array}$ & $20.3(0.3)^{*}$ & $27.3(4.3)$ & 25.3(5.3) & $-{ }^{b}$ & $5.7(1.2)$ & $8.3(2.3)$ & $7.0(3.1)$ & $-b$ \\
\hline
\end{tabular}


Percent Larger-Reinforcer Choice. Figure 1 shows percent larger-reinforcer choice averaged across subjects in the delay-added and delay-subtracted groups for the last five sessions (filled circles) during the 0-s (top-left panel, a), 2-s (top-right panel, b), 4-s (bottom-left panel, c), and 8-s (bottom-right panel, d) delay conditions averaged across blocks of trials. These data were averaged across subjects in the delay-added and delay-subtracted groups because there was no significant interaction between group and delay block during any delay condition with percent larger-reinforcer choice as the dependent measure. Data in Figure 1 were plotted across blocks of trials rather than actual delay values because the functionally determined larger-reinforcer delay series was not the same for all subjects. The functionally determined delay series for subjects in the delay-added group during the 0 -s delay condition was $0,10,20,40,60$ s for CK1, CK-3, CK-5, and CK-6, and 0, 5, 10, 20, 40 s for CK-7. For subjects in the delay-subtracted group the functionally determined delay series during the 4-s delay condition was 4, 14, 24, 44, 64 s for CK-2, CK-4, and CK-8. For all subjects, regardless of delay condition, delay discounting was observed in that percent larger-reinforcer choice decreased as the delay to the larger reinforcer increased across blocks of trials. This result was confirmed by a significant main effect of delay block during each delay condition, [0-s delay condition, $F(4,24)=54.37, p$ $<.001$; 2-s delay condition, $F(4,24)=44.90, p<.001$; 4-s delay condition, $F(4,24)=35.53, p<$ .001 ; 8-s delay condition, $F(4,4)=49.81, p=.001]$. Percent larger-reinforcer choice in each block of trials for individual subjects during each delay condition is shown in Table 2.

Data from the last probe session conducted during each delay condition are also shown in each panel of Figure 1 (unfilled circles). Percent larger-reinforcer choice during the last probe session, averaged across blocks of trials for subjects in the delay-added and delay-subtracted 

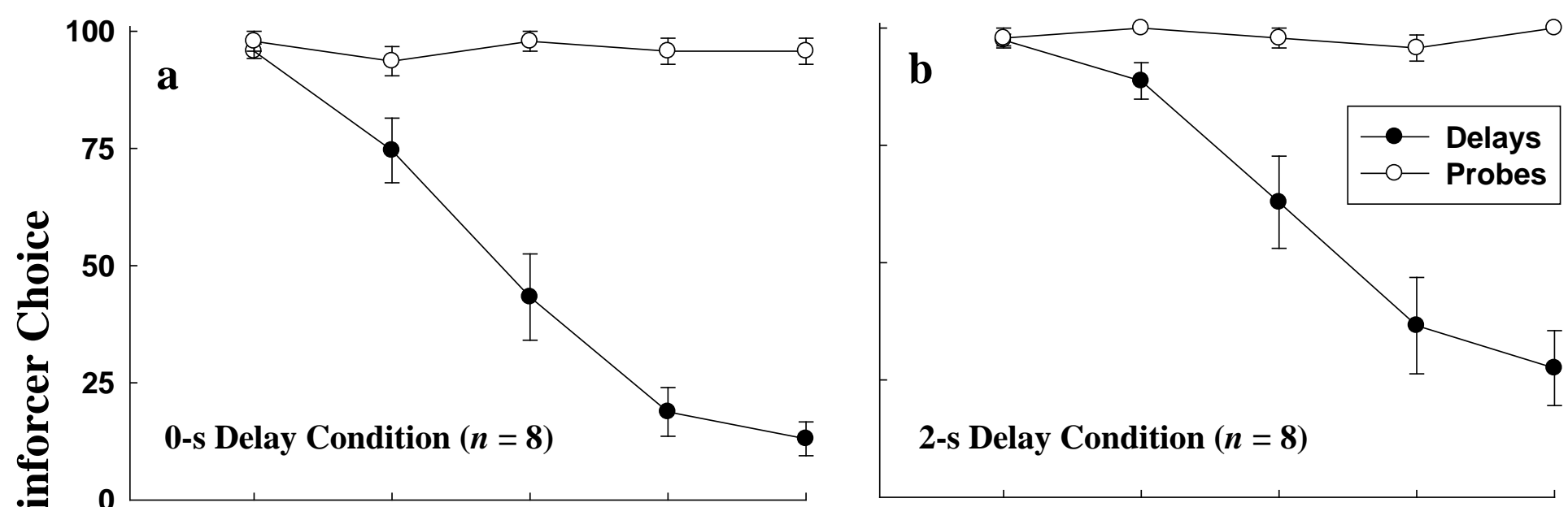

2-s Delay Condition $(n=8)$

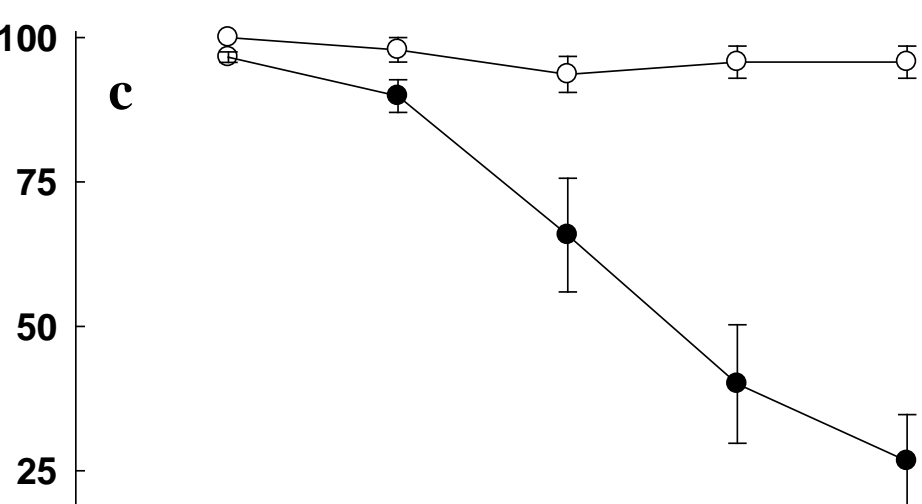

4-s Delay Condition $(n=8)$

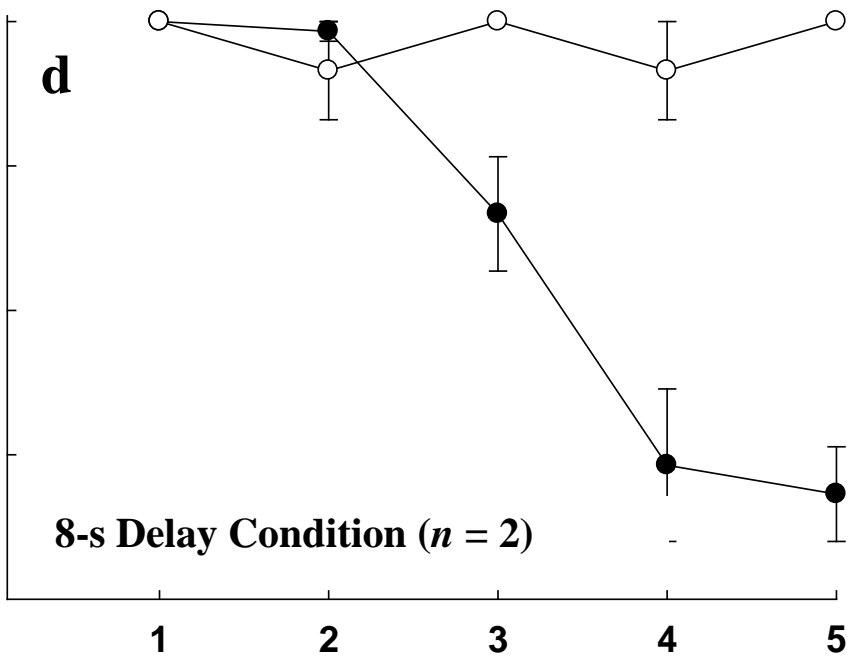

\section{Block of Trials}

Figure 1. Percent larger-reinforcer choice averaged across subjects in the delay-added and delay-subtracted groups for the last five sessions (filled circles) and for the last probe session conducted (unfilled circle) during the 0-s (top-left panel, a), 2-s (top-right panel, b), 4-s (bottomleft panel, c), and 8-s (bottom-right panel, d) delay conditions as a function of block of trials. Error bars are \pm 1 SEM. The delay condition and number of subjects exposed to that delay condition are shown above the $\mathrm{x}$-axis in each panel. Only CK-1 and CK-7 were exposed to the 8-s delay condition. 
groups was $96 \%(S E M=2)$ for the 0-s delay condition, $98 \%(S E M=1)$ for the 2-s delay condition, $97 \%(S E M=1)$ for the 4-s delay condition, and 97\% $(S E M=3 \%)$ for the 8-s delay condition. During the last probe session there was no significant interaction or main effect of delay condition with percent larger-reinforcer choice as the dependent measure. These data are representative of data from individual subjects in the delay-added and delay-subtracted groups and they indicate that control by reinforcer amount was maintained during each delay condition in that percent larger-reinforcer choice across all blocks of free-choice trials was at least $80 \%$.

Area Under the Curve. Figure 2 shows AUC from the last five sessions averaged across subjects in the delay-added and delay-subtracted groups during each delay condition. These data were averaged across groups because there was no significant interaction between delay group and delay condition with AUC as the dependent measure; however, there was a main effect of delay condition, $F(2,12)=5.01, p=.026$. Specifically, AUC was significantly smaller during the 0 -s delay condition $(M=0.39, S E M=0.05)$ compared the 2-s delay condition $(M=$ $0.55, S E M=0.08)$ and 4-s delay condition $(M=0.57, S E M=0.08),[t(7)=2.42, p=.046 ; t(7)=$ 2.74, $p=.029$, respectively]. There was no significant difference in AUC during the 2-s delay condition and the 4-s delay condition.

Additional analyses (two-sample $\mathrm{t}$ tests) were conducted for CK-1 and CK-7 because these were the only subjects exposed to the 8-s delay condition. For CK-1, AUC was significantly smaller during the 0 -s $(M=0.49, S E M=0.03)$, 2-s $(M=0.48$, SEM $=0.02)$, and 4-s $(M=0.46, S E M=0.03)$ delay conditions compared to the 8-s delay condition $(M=0.61, S E M=$ 0.03), $[t(8)=2.93, p=.018 ; t(8)=3.33, p=.01 ; t(8)=3.53, p=.007$, respectively]. For CK-7, AUC was significantly smaller during the 0 -s $(M=0.28$, SEM $=0.02)$, 2-s $(M=0.21, S E M=$ $0.01)$, and 4-s $(M=0.21, S E M=0.01)$ delay conditions compared to the 8-s delay condition $(M=$ 
0.35, SEM = 0.03), $[t(8)=2.35, p=.046 ; t(8)=5.32, p<.001 ; t(8)=5.28, p<.001$, respectively]. AUC for CK-7 was also significantly smaller during the 2-s and 4-s delay conditions compared to the 0 -s delay condition, $[t(8)=4.03, p=.004 ; t(8)=4.01, p=.004$, respectively]. Because of the variability in AUC across subjects during each delay condition, AUC for individual subjects are also plotted in Figure 2 and shown in Table 2.

Although different levels of impulsive choice were generated by adding or subtracting delays common to both reinforcers, there was no significant difference in AUC during the final delay condition prior to $d$-amphetamine administration for subjects in the delay-added group ( $M$ $=0.61, S E M=0.09)$ compared to subjects in the delay-subtracted group $(M=0.47, S E M=0.14)$. This result was due to the relatively small AUC for CK-7 $(M=0.35, S E M=0.03)$ in the delayadded group and the relatively large AUC for CK-6 $(M=0.87, S E M=0.03)$ in the delaysubtracted group. Note that data from CK-6 in these analyses were obtained during the 0-s delay condition before it was re-exposed to the delay conditions. AUC during the final delay condition was therefore used to rank order subjects into either a High-impulsive group (the four subjects with the smallest AUC) or Low-impulsive group (the four subjects with the largest AUC). Figure 3 shows AUC during the final delay condition for subjects in the High-impulsive group (CK-2, CK-4, CK-7, and CK-8) and the Low-impulsive group (CK-1, CK-3, CK-5, and CK-6). A two-sample t test indicated that AUC was significantly smaller during the final delay condition for subjects in the High-impulsive group $(M=0.34, S E M=0.05)$ compared to subjects in the Low-impulsive group $(M=0.75, S E M=0.05), t(6)=5.82, p=.001$, indicating that subjects in the High-impulsive group had higher levels of impulsive choice than subjects in the Lowimpulsive group. 
Table 2

Mean percent larger-reinforcer choice in each block of trials and area under the curve (AUC) averaged across the last five sessions for each delay condition with standard error of the means in parentheses for individual subjects in the delay-added (A) or delay-subtracted (S) groups.

\begin{tabular}{|c|c|c|c|c|c|c|c|}
\hline \multirow{2}{*}{ Subject } & \multirow{2}{*}{$\begin{array}{c}\text { Delay } \\
\text { Condition }\end{array}$} & \multicolumn{5}{|c|}{ Blocks of Trials } & \multirow{2}{*}{ AUC } \\
\hline & & $\underline{1}$ & $\underline{2}$ & $\underline{3}$ & $\underline{4}$ & $\underline{5}$ & \\
\hline \multicolumn{8}{|l|}{ CK-1 (A) } \\
\hline & $0 \mathrm{~s}$ & $93(4)$ & $83(13)$ & $53(6)$ & $30(6)$ & $24(7)$ & $0.49(0.03)$ \\
\hline & $2 \mathrm{~s}$ & $90(4)$ & $80(6)$ & 47 (6) & $30(3)$ & $33(5)$ & $0.48(0.02)$ \\
\hline & $4 \mathrm{~s}$ & $97(3)$ & 77 (11) & $56(11)$ & $23(4)$ & $17(7)$ & $0.46(0.03)$ \\
\hline & $8 \mathrm{~s}$ & $100(0)$ & $100(0)$ & 77 (9) & $36(3)$ & $26(7)$ & $0.61(0.03)$ \\
\hline \multicolumn{8}{|l|}{ CK-3 (A) } \\
\hline & $0 \mathrm{~s}$ & 97 (3) & $43(7)$ & $23(7)$ & $10(7)$ & $7(4)$ & $0.26(0.02)$ \\
\hline & $2 \mathrm{~s}$ & $100(0)$ & $97(3)$ & $83(11)$ & $47(8)$ & $33(5)$ & $0.66(0.04)$ \\
\hline & $4 \mathrm{~s}$ & $93(4)$ & $93(4)$ & $83(9)$ & $53(8)$ & $30(6)$ & $0.67(0.04)$ \\
\hline \multicolumn{8}{|l|}{ CK-5 (A) } \\
\hline & $0 \mathrm{~s}$ & $100(0)$ & $100(0)$ & $77(7)$ & $47(8)$ & $30(10)$ & $0.65(0.03)$ \\
\hline & $2 \mathrm{~s}$ & 97 (3) & 97 (3) & $86(3)$ & $67(12)$ & $40(11)$ & $0.75(0.05)$ \\
\hline & $4 \mathrm{~s}$ & $100(0)$ & $100(0)$ & $97(3)$ & $63(10)$ & $53(10)$ & $0.79(0.05)$ \\
\hline \multicolumn{8}{|l|}{ CK-6 (A) $)^{\mathrm{a}}$} \\
\hline & $0 \mathrm{~s}$ & $100(0)$ & $73(4)$ & $57(7)$ & $17(5)$ & $17(5)$ & $0.43(0.01)$ \\
\hline & $2 \mathrm{~s}$ & $100(0)$ & $90(7)$ & $83(7)$ & $86(3)$ & $70(6)$ & $0.85(0.02)$ \\
\hline & $4 \mathrm{~s}$ & $100(0)$ & $100(0)$ & $87(6)$ & $87(6)$ & $67(7)$ & $0.87(0.03)$ \\
\hline \multicolumn{8}{|l|}{ CK-7 (A) } \\
\hline & $0 \mathrm{~s}$ & $90(4)$ & $80(6)$ & $20(6)$ & $17(5)$ & $10(4)$ & $0.28(0.02)$ \\
\hline & $2 \mathrm{~s}$ & 97 (3) & $67(11)$ & $17(7)$ & $7(4)$ & $3(3)$ & $0.21(0.01)$ \\
\hline & $4 \mathrm{~s}$ & 97 (3) & $83(9)$ & $10(4)$ & $3(3)$ & $7(4)$ & $0.21(0.01)$ \\
\hline & $8 \mathrm{~s}$ & $100(0)$ & 97 (3) & $57(7)$ & $10(7)$ & $10(10)$ & $0.35(0.03)$ \\
\hline \multicolumn{8}{|l|}{ CK-2 (S) } \\
\hline & $0 \mathrm{~s}$ & 97 (3) & $63(6)$ & 30 (3) & $23(4)$ & $13(6)$ & $0.36(0.03)$ \\
\hline & $2 \mathrm{~s}$ & $100(0)$ & $100(0)$ & $83(7)$ & $36(3)$ & $27(4)$ & $0.65(0.03)$ \\
\hline & $4 \mathrm{~s}$ & 97 (3) & $90(4)$ & $67(7)$ & 40 (11) & 17 (9) & $0.60(0.02)$ \\
\hline \multicolumn{8}{|l|}{ CK-4 (S) } \\
\hline & $0 \mathrm{~s}$ & $100(0)$ & $57(4)$ & $10(7)$ & $0(0)$ & $0(0)$ & $0.20(0.02)$ \\
\hline & $2 \mathrm{~s}$ & 97 (3) & 87 (6) & $30(6)$ & $3(3)$ & $0(0)$ & $0.31(0.02)$ \\
\hline & $4 \mathrm{~s}$ & 97 (3) & $86(3)$ & 47 (8) & $3(3)$ & $0(0)$ & $0.35(0.03)$ \\
\hline \multicolumn{8}{|l|}{ CK-8 (S) } \\
\hline & $0 \mathrm{~s}$ & $90(4)$ & 97 (3) & 77 (13) & 7 (7) & $4(3)$ & $0.46(0.04)$ \\
\hline & $2 \mathrm{~s}$ & $100(0)$ & $93(7)$ & $73(10)$ & $17(7)$ & $14(3)$ & $0.50(0.03)$ \\
\hline & $4 \mathrm{~s}$ & $93(4)$ & $90(4)$ & $80(10)$ & 47 (6) & $23(13)$ & $0.62(0.05)$ \\
\hline
\end{tabular}

$-{ }^{\mathrm{a}}$ Based on data obtained during re-exposure to delay conditions. 


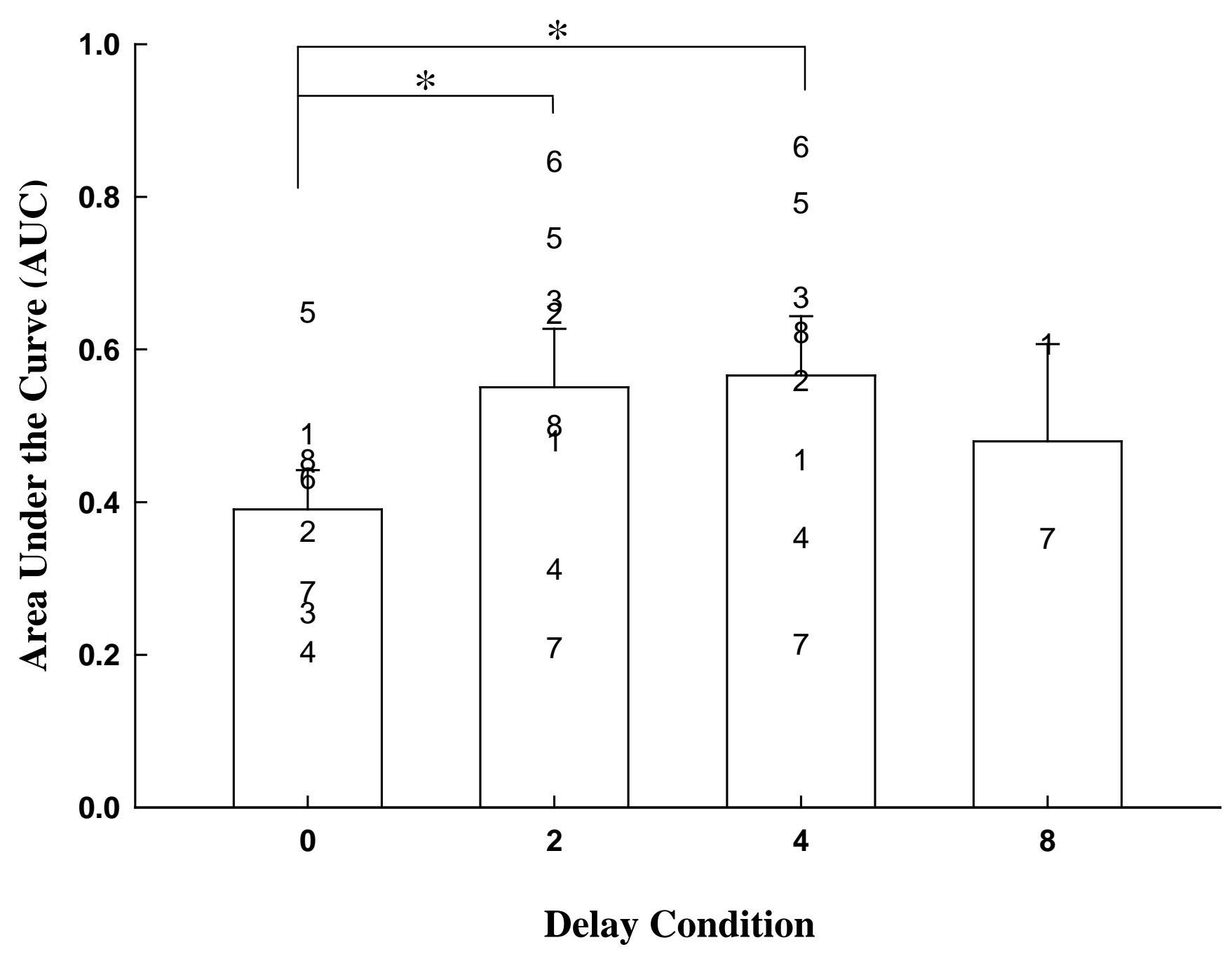

Figure 2. Area under the curve (AUC) averaged across subjects in the delay-added and delaysubtracted groups for the last five sessions during each delay condition. Error bars are \pm 1 SEM. Individual subject data are represented by the respective subject number (i.e., CK-1 $=1$ ). Only CK-1 and CK-7 were exposed to the 8-s delay condition. Asterisks represent differences in AUC that were significant at $p<.05$. 


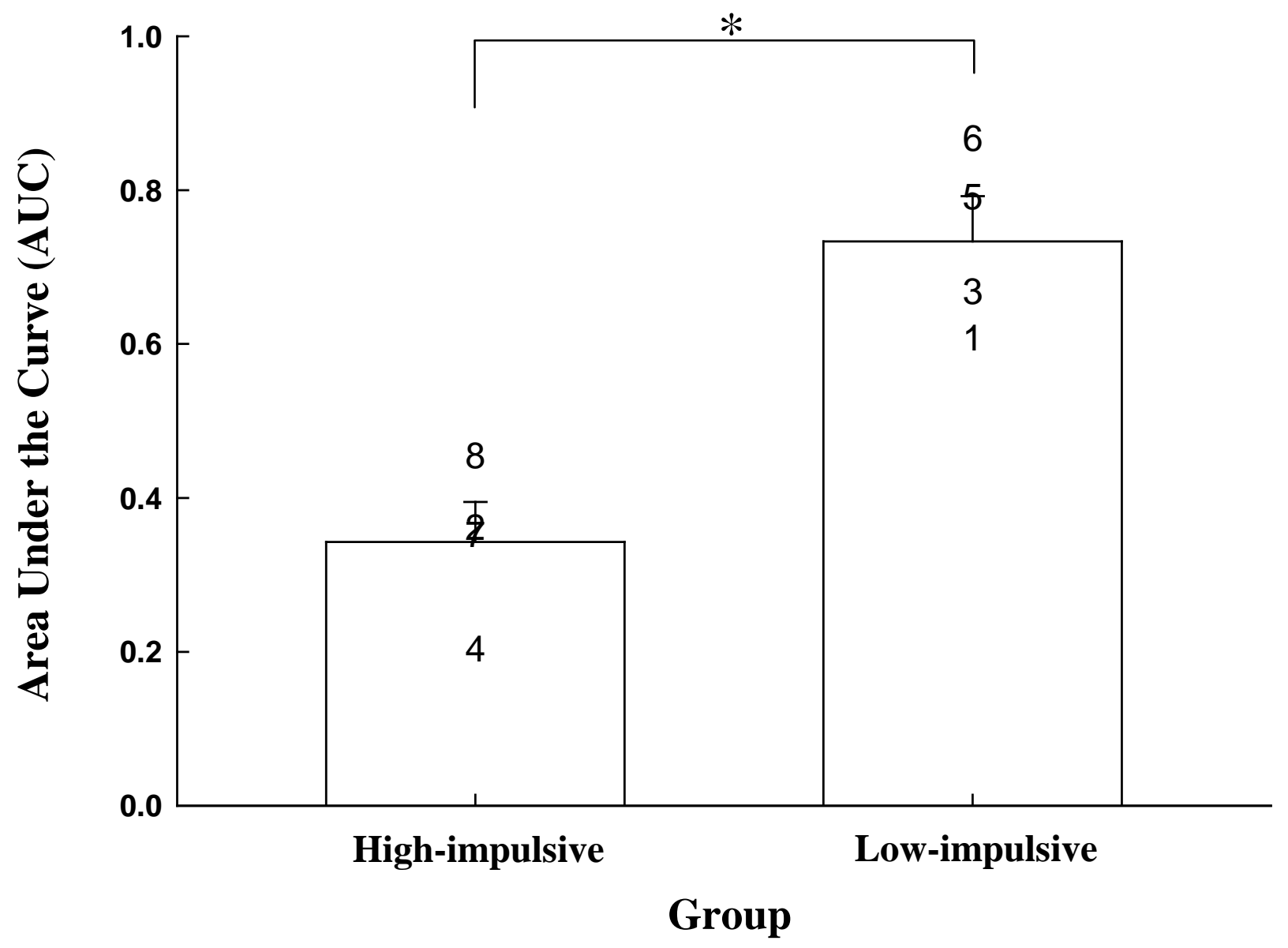

Figure 3. Area under the curve (AUC) for subjects in the High-impulsive and Low-impulsive groups during the final delay condition. Error bars are \pm 1 SEM. Individual subject data are represented by the respective subject numbers (i.e., CK-1 = 1). Data for CK-2 and CK-7 (Highimpulsive group) overlap and data for CK-6 (Low-impulsive group) were obtained before reexposure to delay conditions. The asterisk represents difference in AUC that was significant at $p$ $<.05$. 


\section{Acute d-Amphetamine Phase}

Table 3 and Table 4 show percent larger-reinforcer choice in each block of trials and AUC for control sessions and each dose of $d$-amphetamine administered for individual subjects in the High-impulsive group and Low-impulsive group, respectively. Different levels of impulsive choice were maintained during the acute $d$-amphetamine phase as two-sample t tests indicated that AUC was significantly smaller for subjects in the High-impulsive group during control sessions $(M=0.32, S E M=0.04)$ and saline administrations $(M=0.32, S E M=0.04)$ compared to subjects in the Low-impulsive group during control sessions $(M=0.78, S E M=$ $0.02)$ and saline administrations $(M=0.77, S E M=0.02),[t(6)=9.85, p<.001 ; t(6)=10.77, p<$ .001 , respectively].

Because the dose and the number of administrations of each dose of $d$-amphetamine differed across subjects, effects of $d$-amphetamine on AUC were analyzed for individual subjects in the High-impulsive group and Low-impulsive group using two-sample t tests; however, these tests were not conducted for doses of $d$-amphetamine that decreased percent larger-reinforcer in the first block of free-choice trials below $80 \%$. To show general trends, however, these data were expressed as percent of saline AUC for individual subjects in the High-impulsive group (Figure 4) and Low-impulsive groups (Figure 5).

High-impulsive Group. Figure 4 shows effects of each dose of $d$-amphetamine administered for individual subjects in the High-impulsive group expressed as percent of saline AUC (indicated by the dashed horizontal line in each panel). Select doses of $d$-amphetamine significantly increased AUC compared to saline for CK-4 (upper-right panel, b) and CK-7 (lower-left panel, c) at $0.03 \mathrm{mg} / \mathrm{kg} d$-amphetamine and for CK-8 (lower-right panel, d) at 1.8 $\mathrm{mg} / \mathrm{kg} d$-amphetamine $[t(6)=2.53, p=.044 ; t(10)=2.23, p=.049 ; t(5)=3.22, p=.02$, 
Table 3.

Mean percent larger-reinforcer choice and area under the curve (AUC) for control sessions and each dose of $d$ amphetamine administered for individual subjects in the High-impulsive group with standard error of the means in parentheses. The number of control sessions and administrations of each dose of d-amphetamine are in parentheses. The larger-reinforcer delay series (s) in effect during d-amphetamine administration is also shown. Asterisks represent differences in AUC compared to saline that were significant at $p<.05$ and crosses represent doses of $d$ amphetamine that decreased percent larger-reinforcer choice during the first block of free-choice trials below 80\%.

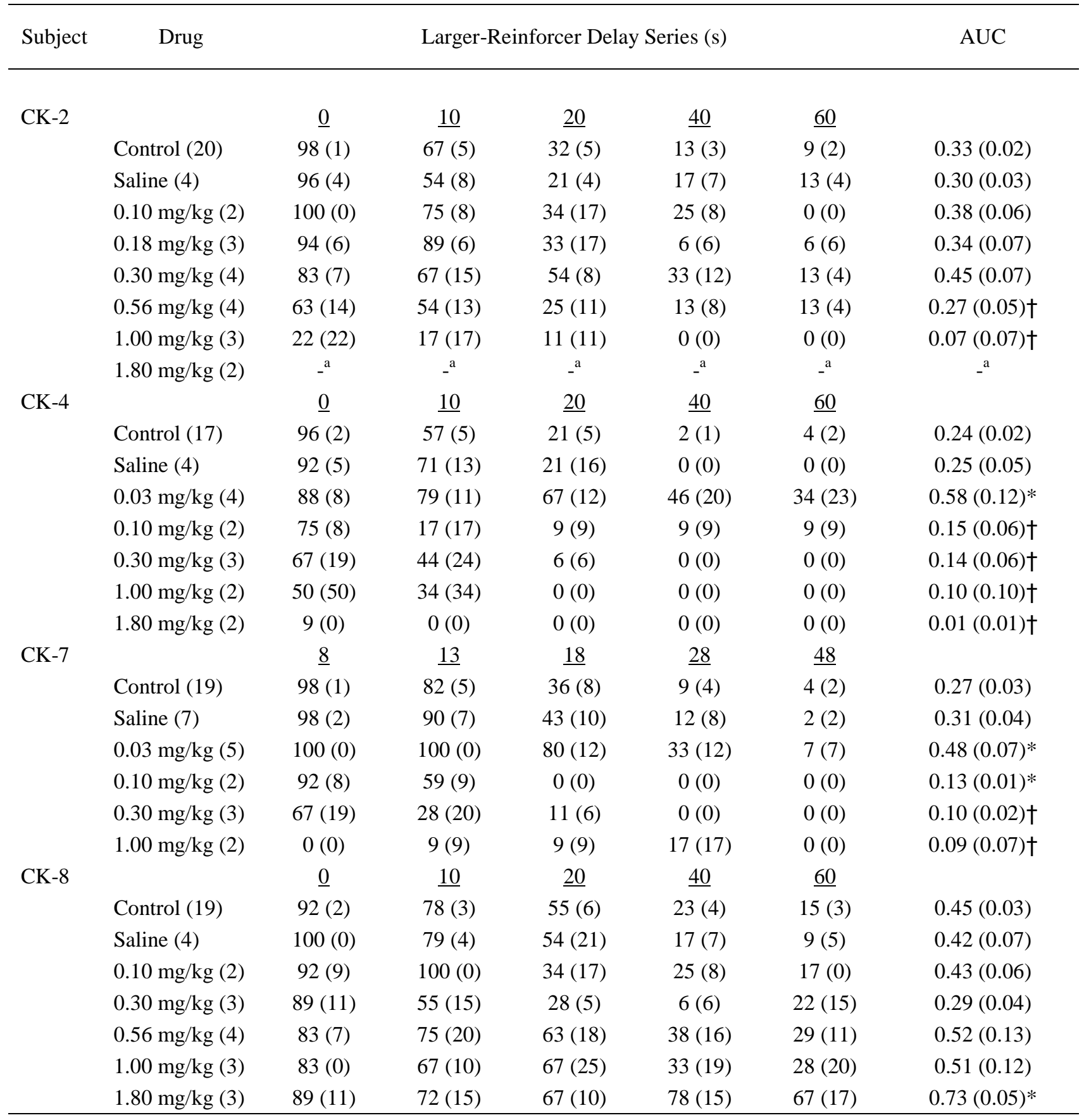

\footnotetext{
- ${ }^{\mathrm{a}}$ Data were not included due to excessive response omissions.
} 


\section{Table 4.}

Mean percent larger-reinforcer choice and area under the curve (AUC) for control sessions and each dose of damphetamine administered for individual subjects in the Low-impulsive group with standard error of the means in parentheses. The number of control sessions and administrations of each dose of d-amphetamine are in parentheses. The larger-reinforcer delay series (s) in effect during d-amphetamine administration is also shown. Asterisks represent differences in AUC compared to saline that were significant at $p<.05$ and crosses represent doses of $d$ amphetamine that decreased percent larger-reinforcer choice during the first block of free-choice trials below $80 \%$.

\begin{tabular}{|c|c|c|c|c|c|c|c|}
\hline \multirow{2}{*}{$\begin{array}{l}\text { Subject } \\
\text { CK-1 }\end{array}$} & \multirow[t]{2}{*}{ Drug } & \multicolumn{5}{|c|}{ Larger-Reinforcer Delay Series (s) } & \multirow[t]{2}{*}{ AUC } \\
\hline & & $\underline{8}$ & $\underline{18}$ & $\underline{28}$ & $\underline{48}$ & $\underline{68}$ & \\
\hline & Control (17) & $100(0)$ & $96(2)$ & $82(5)$ & $63(4)$ & $47(6)$ & $0.73(0.03)$ \\
\hline & Saline (3) & $100(0)$ & $94(6)$ & $83(10)$ & $55(15)$ & $50(10)$ & $0.72(0.09)$ \\
\hline & $0.10 \mathrm{mg} / \mathrm{kg}(3)$ & $94(6)$ & $83(0)$ & $72(15)$ & $72(5)$ & $55(15)$ & $0.73(0.01)$ \\
\hline & $0.30 \mathrm{mg} / \mathrm{kg}(5)$ & $808(8)$ & $70(3)$ & $63(13)$ & $77(7)$ & $40(9)$ & $0.66(0.06) \dagger$ \\
\hline & $1.00 \mathrm{mg} / \mathrm{kg}(3)$ & $78(5)$ & $67(16)$ & $56(50)$ & $50(17)$ & $44(20)$ & $0.55(0.05) \dagger$ \\
\hline & $1.80 \mathrm{mg} / \mathrm{kg}(3)$ & $61(11)$ & $78(5)$ & $50(10)$ & $39(15)$ & $66(17)$ & $0.55(0.04) \dagger$ \\
\hline \multirow[t]{7}{*}{ CK-3 } & & $\underline{4}$ & $\underline{14}$ & $\underline{24}$ & $\underline{44}$ & $\underline{64}$ & \\
\hline & Control (15) & $100(0)$ & $97(2)$ & $94(2)$ & $77(4)$ & $40(6)$ & $0.80(0.02)$ \\
\hline & Saline (3) & $100(0)$ & $100(0)$ & $94(6)$ & $72(15)$ & $50(10)$ & $0.81(0.07)$ \\
\hline & $0.10 \mathrm{mg} / \mathrm{kg}(2)$ & $100(0)$ & $100(0)$ & $100(0)$ & $75(0)$ & 34 (17) & $0.81(0.05)$ \\
\hline & $0.30 \mathrm{mg} / \mathrm{kg}(4)$ & $83(7)$ & $96(4)$ & $87(4)$ & $71(14)$ & $50(22)$ & $0.77(0.05)$ \\
\hline & $1.00 \mathrm{mg} / \mathrm{kg}$ (3) & $83(10)$ & $72(5)$ & $50(17)$ & $33(10)$ & 39 (11) & $0.49(0.04)^{*}$ \\
\hline & $1.80 \mathrm{mg} / \mathrm{kg}$ (3) & $39(24)$ & $28(20)$ & $11(11)$ & $22(5)$ & $17(17)$ & $0.21(0.13) \dagger$ \\
\hline \multirow[t]{8}{*}{ CK-5 } & & $\underline{4}$ & $\underline{14}$ & $\underline{24}$ & $\underline{44}$ & $\underline{64}$ & \\
\hline & Control (20) & $100(0)$ & $99(0)$ & $94(3)$ & $72(5)$ & $58(4)$ & $0.82(0.03)$ \\
\hline & Saline (3) & $100(0)$ & $100(0)$ & $89(11)$ & $61(6)$ & $50(10)$ & $0.76(0.06)$ \\
\hline & $0.03 \mathrm{mg} / \mathrm{kg}(2)$ & $100(0)$ & $100(0)$ & $92(9)$ & $84(17)$ & $59(9)$ & $0.86(0.06)$ \\
\hline & $0.10 \mathrm{mg} / \mathrm{kg}$ (3) & $94(6)$ & $94(6)$ & $94(6)$ & $83(10)$ & $50(10)$ & $0.83(0.05)$ \\
\hline & $0.30 \mathrm{mg} / \mathrm{kg}(3)$ & $100(0)$ & $100(0)$ & $89(6)$ & $89(6)$ & 89 (11) & $0.92(0.04)$ \\
\hline & $1.00 \mathrm{mg} / \mathrm{kg}(6)$ & $81(8)$ & $92(6)$ & $81(8)$ & $69(7)$ & $47(12)$ & $0.73(0.06)$ \\
\hline & $1.80 \mathrm{mg} / \mathrm{kg}$ (3) & $56(6)$ & $44(6)$ & $44(11)$ & $78(15)$ & 67 (19) & $0.60(0.10) \dagger$ \\
\hline \multirow[t]{9}{*}{ CK-6 ${ }^{\mathrm{b}}$} & & $\underline{0}$ & 10 & 20 & 40 & $\underline{60}$ & \\
\hline & Control (26) & $100(0)$ & $99(1)$ & $95(2)$ & $68(4)$ & $38(4)$ & $0.78(0.01)$ \\
\hline & Saline (6) & $97(3)$ & $95(6)$ & $89(8)$ & $72(7)$ & 45 (11) & $0.78(0.04)$ \\
\hline & $0.03 \mathrm{mg} / \mathrm{kg}(4)$ & $96(4)$ & $100(0)$ & $92(8)$ & $46(11)$ & $38(8)$ & $0.69(0.05)$ \\
\hline & $0.10 \mathrm{mg} / \mathrm{kg}$ (3) & $94(6)$ & $94(6)$ & $94(6)$ & $50(10)$ & $28(11)$ & $0.69(0.03)$ \\
\hline & $0.30 \mathrm{mg} / \mathrm{kg}(3)$ & $94(6)$ & $100(0)$ & $89(6)$ & $56(6)$ & $33(10)$ & $0.71(0.02)$ \\
\hline & $0.56 \mathrm{mg} / \mathrm{kg}(6)$ & $45(16)$ & $67(14)$ & $78(10)$ & $50(9)$ & $47(8)$ & $0.59(0.07) \dagger$ \\
\hline & $1.00 \mathrm{mg} / \mathrm{kg}(4)$ & $67(12)$ & $29(4)$ & $21(8)$ & $54(11)$ & $29(13)$ & $0.36(0.06) \dagger$ \\
\hline & $1.80 \mathrm{mg} / \mathrm{kg}(1)$ & $-{ }^{\mathrm{a}}$ & $-{ }^{\mathrm{a}}$ & $-{ }^{\mathrm{a}}$ & $-^{\mathrm{a}}$ & $-{ }^{\mathrm{a}}$ & $-^{\mathrm{a}}$ \\
\hline
\end{tabular}

$-^{\mathrm{a}}$ Data were not included due to excessive response omissions.

- ${ }^{\mathrm{b}}$ Data were obtained prior to re-exposure to delay conditions. 
respectively]. For CK-2 (upper-left panel, a) the increase in AUC at $0.3 \mathrm{mg} / \mathrm{kg} d$-amphetamine compared to saline was not significant. For CK-7 there was also a significant decrease in AUC compared to saline at $0.1 \mathrm{mg} / \mathrm{kg} d$-amphetamine, $t(6)=3.95, p=.007$. No other dose of $d$ amphetamine in which percent larger-reinforcer choice during the first block of free-choice trials was at least $80 \%$ had a significant effect on AUC compared to saline.

Low-impulsive Group. Figure 5 shows effects of each dose of $d$-amphetamine administered for individual subjects in the Low-impulsive group expressed as percent of saline AUC (indicated by the dashed horizontal line in each panel). There were no significant effects of $d$-amphetamine on AUC compared to saline for CK-1 (upper-left panel, d), CK-5 (lower-left panel, c), or CK-6 (lower-right panel, d). For CK-3 (upper-right panel, b), however, $1.0 \mathrm{mg} / \mathrm{kg}$ $d$-amphetamine significantly decreased AUC compared to saline, $t(4)=4.03, p=.015$. No other dose of $d$-amphetamine in which percent larger-reinforcer choice during the first block of freechoice trials was at least $80 \%$ had a significant effect on AUC compared to saline.

\section{Summary}

Different levels of impulsive choice were generated by adding or subtracting delays common to both reinforcers. Adding delays common to both reinforcers decreased impulsive choice (i.e., increased AUC) whereas subtracting delays common to both reinforcers increased impulsive choice (i.e., decreased AUC). During the final delay condition, subjects in the Highimpulsive group had higher levels of impulsive choice compared to subjects in the Lowimpulsive group and effects of $d$-amphetamine generally depended upon these group differences. Select doses of $d$-amphetamine decreased impulsive choice for three of the four subjects in the High-impulsive group but not for any of the subjects in the Low-impulsive group. Although larger doses of $d$-amphetamine tended to increase impulsive choice for all subjects except CK-8, 

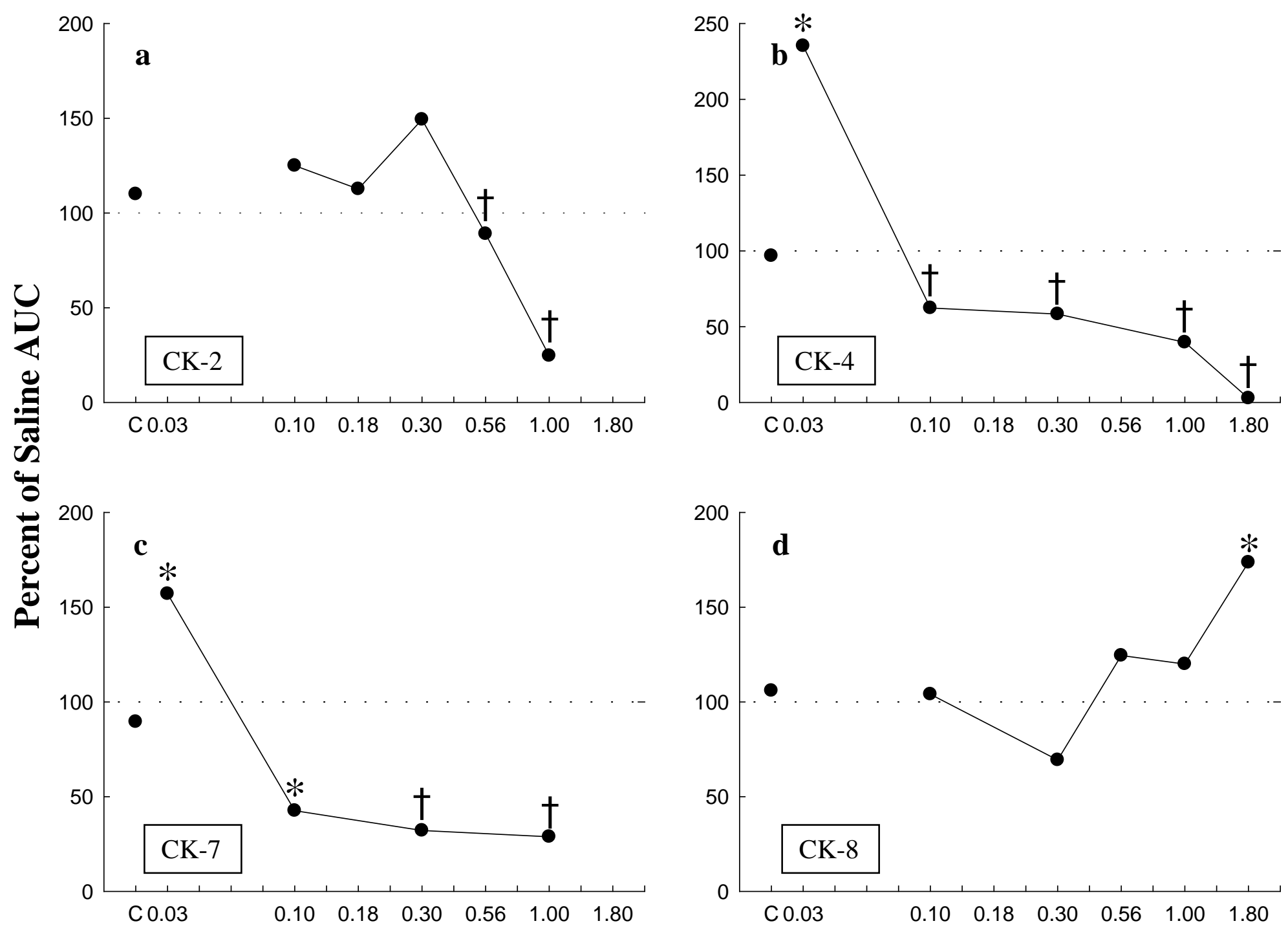

\section{d-Amphetamine (mg/kg)}

Figure 4. Percent of saline AUC (dashed horizontal lines in each panel) for subjects in the Highimpulsive group for control sessions (C) and each dose of $d$-amphetamine administered.

Subjects are identified above the $x$-axis in each panel. The $y$-axis is scaled differently for CK-4. Asterisks represent doses of $d$-amphetamine that changed AUC compared to saline that were significant at $p<.05$ and crosses represent doses that decreased percent larger-reinforcer choice during the first block of free-choice trials below $80 \%$. 


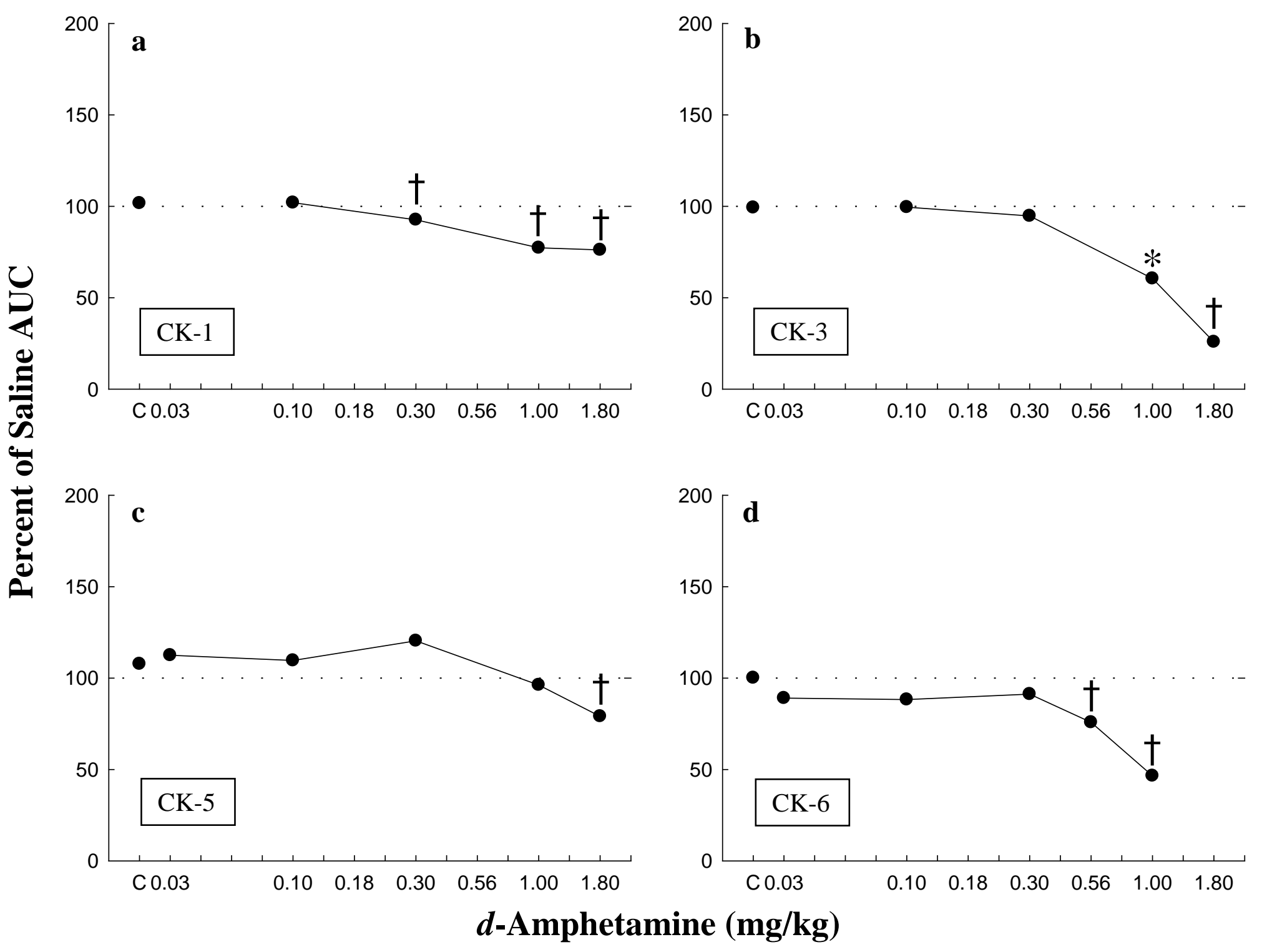

Figure 5. Percent of saline AUC (dashed horizontal lines in each panel) for subjects in the Lowimpulsive group for control sessions (C) and each dose of $d$-amphetamine administered. Subjects are identified above the $x$-axis in each panel. The asterisk represent a dose of $d$ amphetamine that changed AUC compared to saline that was significant at $p<.05$ and crosses represent doses that decreased percent larger-reinforcer choice during the first block of freechoice trials below $80 \%$. 
these effects are difficult to interpret because percent larger-reinforcer choice during the first block of free-choice trials decreased to below $80 \%$.

\section{Discussion}

There were two major findings in the present study. First, different levels of impulsive choice were generated by changing the delay common to both reinforcers. Adding delays common to both reinforcers decreased impulsive choice whereas subtracting delays common to both reinforcers increased impulsive choice. These results extend past research (e.g., Ainslie \& Herrnstein, 1981; Green \& Estle, 2003) by showing that impulsive choice can be changed by adding or subtracting delays common to both reinforcers using a within-session delaydiscounting procedure. Second, effects of $d$-amphetamine on impulsive choice generally depended on the differences in impulsive choice generated by adding or subtracting delays common to both reinforcers. Select doses of $d$-amphetamine decreased impulsive choice for three of the four subjects in the High-impulsive group and either had no effect or increased impulsive choice for subjects in the Low-impulsive group. These results are consistent with past research (e.g., Barbelivien, Billy, Lazarus, Kelche, Majchrzak, 2007; Hand, Fox, \& Reilly, 2009; Perry, Stairs, \& Bardo, 2008) and add further support to the hypothesis that effects of stimulant drugs on impulsive choice depend, at least in part, on baseline levels of impulsive choice.

\section{Delay Discounting}

Percent larger-reinforcer choice decreased as the delay to the larger reinforcer increased across blocks of trials for all subjects. This is consistent with past research (e.g., Anderson \& Diller, 2010; Evenden \& Ryan, 1996) and occurred regardless of whether delays common to both reinforcers were added to or subtracted to the delays in effect. Furthermore, adding delays common to both reinforcers decreased impulsive choice whereas subtracting delays common to 
both reinforcers increased impulsive choice. These results suggest that the delay common to both reinforcers is an important determinant of impulsive choice and provide additional support for the interpretation of impulsive choice being context dependent (cf. Odum \& Baumann, 2010).

One difference between the delay-added and delay-subtracted groups in the present experiment was that subjects in the delay-added group required more sessions to meet stability during the 0 -s delay condition than subjects in the delay-subtracted group. This difference was primarily due to two outliers in the delay-added group but the order of delay condition presentation could have contributed as there was a general decrease in the number of sessions required to meet stability across delay conditions for several subjects in both groups.

Although subjects in the delay-added group required more sessions to meet stability during the 0 -s delay condition than subjects in the delay-subtracted groups there were no differences in impulsive choice between these groups during any delay condition. Collapsing data across groups revealed that levels of impulsive choice were higher during the 0 -s delay condition compared to the other delay conditions for all subjects. For the majority of subjects, impulsive choice systematically decreased as the delay common to both reinforcers increased. For some subjects, however, these effects were less consistent: impulsive choice increased when the delay common to both reinforcers increased (see 2-s and 4-s delay conditions for CK-1 and CK-7) and decreased when the delay common to both reinforcers decreased (see 2-s delay condition for CK-2). Although these changes in impulsive choice were not predicted, impulsive choice did change in the predicted direction during subsequent delay conditions. Similar inconsistencies were reported by Ainslie and Herrnstein (1981) for some subjects when delays common to both reinforcers were subtracted and the absolute difference between the delay conditions was small. The inconsistencies noted above may therefore have been due to the 
relatively small difference between the delay condition values (i.e., 0 s, 2 s, 4 s, 8 s) which were used to keep trial length consistent across subjects and to ensure that drugs administered remained behaviorally active for the duration of each experimental session. Future research might address this issue by using larger, more discrepant delay values or by differentially signaling the delay common to both reinforcers.

Prior to $d$-amphetamine administration subjects rank ordered (based on AUC during the final delay condition) into the High-impulsive group had higher levels of impulsive choice than subjects in the Low-impulsive group. Importantly, this difference was determined primarily by whether subjects were in the delay-added or delay-subtracted group as three of the four subjects in the High-impulsive group were from the delay-subtracted group and three of the four subjects in the Low-impulsive group were from the delay-added group. For all subjects, however, levels of impulsive choice increased as the delay common to both reinforcers increased which suggests that changing the delay common to both reinforcers may be one way to generate different levels of impulsive choice both within and between subjects.

\section{Effects of $d$-Amphetamine}

In the laboratory, effects of stimulant drugs on impulsive choice have been mixed. Stimulant drugs have been reported to decrease impulsive choice (e.g., Diller, Saunders, \& Anderson, 2008; Pitts \& McKinney, 2005) or increase impulsive choice (e.g., Evenden \& Ryan, 1996; Slezak \& Anderson, 2009). In the present experiment, during the final delay condition prior to $d$-amphetamine administration, subjects in the High-impulsive group had higher levels of impulsive choice than subjects in the Low-impulsive group and effects of $d$-amphetamine generally depended on these differences. Select doses of $d$-amphetamine decreased impulsive choice for subjects in the High-impulsive group but not for subjects in the Low-impulsive group. 
These baseline-dependent effects of $d$-amphetamine on impulsive choice are consistent with past research (e.g., Barbelivien, Billy, Lazarus, Kelche, \& Majchrzak, 2007; Fox, Hand, \& Reilly, 2009; Perry, Stairs, \& Bardo, 2008). Barbelivien et al., for example, used a similar withinsession delay-discounting procedure and reported that $d$-amphetamine decreased impulsive choice for rats with medium levels of impulsive choice but had no effects on impulsive choice for rats with low or high levels of impulsive choice. Barbelivien et al.’s results are similar to those of the current experiment in that $d$-amphetamine decreased impulsive choice for subjects with one level of impulsive choice but had no effect on impulsive choice for subjects with a different level of impulsive choice. Despite these similarities, direct comparisons between the findings reported by Barbelivien et al. and the current experiment are limited in that the current study altered the delay common to both reinforcers to generate different levels of impulsive choice whereas Barbelivien et al. used a large sample size and different technique to classify baseline levels of impulsive choice. Despite these procedural differences, results from Barbelivien et al. and the current experiment show that $d$-amphetamine differentially affected impulsive choice for subjects with different levels of impulsive choice and thus add further support for the hypothesis that effects of stimulant drugs depend, at least in part, on baseline levels of impulsive choice.

Although the decrease in impulsive choice following administration of select doses of $d$ amphetamine appeared to depend on baseline levels of impulsive choice, these effects could be due to other factors such as response perseveration on the lever associated with the larger reinforcer. This is unlikely, however, because response perseveration would have been equally likely to occur on the lever associated with the smaller reinforcer as the order in which the choice alternatives were presented during forced-exposure trials (which preceded free-choice trials) was 
random. In addition, percent larger-reinforcer choice decreased as the delay to the larger reinforcer increased, even at the doses of $d$-amphetamine that decreased impulsive choice. There are also some potential reasons for why $d$-amphetamine did not affect impulsive choice for subjects in the Low-impulsive group. First, percent larger-reinforcer choice for subjects in the Low-impulsive group was already relatively high across delay blocks. Therefore, $d$-amphetamine could only increase percent larger-reinforcer choice by a small amount before choice for the larger reinforcer was exclusive. Second, although there appeared to be a dosedependent increase in impulsive choice for subjects in the Low-impulsive group, the doses of $d$ amphetamine that increased impulsive choice also decreased percent larger-reinforcer choice in the first block of free-choice trials below $80 \%$. This makes it difficult to determine if effects of $d$-amphetamine on impulsive choice were due to a disruption in control by reinforcer amount, delay, or some combination of both (cf. Pitts \& Febbo, 2004). Overall, results from the present experiment indicate that effects of $d$-amphetamine depended on different baseline levels of impulsive choice; however, the factors responsible for this selective effect of $d$-amphetamine are not clear and require further research.

Future research could evaluate effects of stimulant drugs on different levels of impulsive choice using a within-subject design. For example, delay discounting could be established and then effects of $d$-amphetamine determined. Across subsequent phases, the delay common to both reinforcers could be altered to generate different levels of impulsive choice and then effects of $d$-amphetamine could be re-determined within subject. It might also be useful to determine how environmental variables such as changing the delay common to both reinforcers interacts with genetic variables known to influence impulsive choice. Certain inbred strains of rats, for example, have been shown to make more impulsive choices than others (e.g., Anderson \& 
Woolverton, 2005; Fox, Hand, Reilly, 2009; Huskinson, Krebs, \& Anderson, 2011; Wilhelm \& Mitchell, 2009) and it would be useful to determine whether environmental manipulations can attenuate these strain differences. Such research might provide additional information about the interaction between environmental and genetic determinants of impulsive choice.

\section{Conclusions}

In summary, impulsive choice decreased by adding delays common to both reinforcers and increased by subtracting delays common to both reinforcers using a within-session delaydiscounting procedure. During the final delay condition prior to $d$-amphetamine administration, subjects in the High-impulsive group had higher levels of impulsive choice than subjects in the Low-impulsive group and effects of $d$-amphetamine generally depended on these differences. Select doses of $d$-amphetamine decreased impulsive choice for subjects in the High-impulsive group but not for subjects in the Low-impulsive group. These results show that different levels of impulsive choice can be generated by changing the delay common to both reinforcers and suggest that effects of $d$-amphetamine on impulsive choice depend, at least in part, on baseline levels of impulsive choice. The current experiment demonstrates how effects of drugs can be influenced by behavioral variables and may potentially provide additional information about the discrepant effects of stimulant drugs on impulsive choice. 


\section{References}

Alessi, S. M., \& Petry, N. M. (2003). Pathological gambling severity is associated with impulsivity in a delay discounting procedure. Behavioural Processes, 64, 345-354.

Anderson, K. G., \& Diller, J. W. (2010). Effects of acute and repeated nicotine administration on delay discounting in Lewis and Fischer 344 rats. Behavioural Pharmacology, 21, 754764.

Anderson, K. G., \& Woolverton, W. L. (2005). Effects of clomipramine on self-control choice in Lewis and Fischer 344 rats. Pharmacology, Biochemistry, \& Behavior, 80, 387-393.

Ainslie, G. W. (1975). Specious reward: A behavioral theory of impulsiveness and impulse control. Psychological Bulletin, 82, 463-496.

Ainslie, G. W., \& Herrnstein, R. J. (1981). Preference reversal and delayed reinforcement. Animal Learning and Behavior, 9, 476-482.

Barbelivien, A., Billy, E., Lazarus, C., Kelche, C., \& Majchrzak, M. (2007). Rats with different profiles of impulsive choice behavior exhibit differences in responses to caffeine and $d$ amphetamine and in medial prefrontal cortex 5-HT utilization. Behavioural Brain Research, 187, 273-283.

Barkley, R. A. (1977). A review of stimulant drug research with hyperactive children. Journal of Child Psychology and Psychiatry, 18, 137-165.

Barkley, R. A., \& Biederman, J. (1997). Toward a broader definition of the age-of-onset criterion for attention-deficit hyperactivity disorder. Journal of American Academic Child Adolescence and Psychiatry, 36, 1204-1210.

Bickel, W. K., Odum, A. L., \& Madden, G. J. (1999). Impulsivity and cigarette smoking: Delay discounting in current, never, and ex-smokers. Psychopharmacology, 146, 447-454. 
Cardinal, R. N., Robbins, T. W., \& Everitt, B. J. (2000). The effects of $d$-amphetamine, chlordiazepoxide, $a$-fluepenthixol, and behavioural manipulations on choice of signalled and unsignalled delayed reinforcement in rats. Psychopharmacology, 152, 362-375.

Charrier, D., \& Thiebot, M. H. (1996). Effects of psychotropic drugs on rats responding in an operant paradigm involving choice between delayed reinforcers. Pharmacology, Biochemistry, and Behavior, 54, 149-157.

Cherek D. R., \& Lane, S. D. (1999). Effects of d,l-fenfluramine on aggressive and impulsive responding in adult males with a history of conduct disorder. Psychopharmacology, 146, 473-481.

Deluty, M. Z. (1978). Self-control and impulsiveness involving aversive events. Journal of Experiments Psychology, 4(3), 250-266.

Diller, J. W., Saunders, B. T., \& Anderson, K. G. (2008). Effects of acute and repeated administration of caffeine on temporal discounting in rats. Pharmacology, Biochemistry and Behavior, 89, 546-555.

Eagle, D. M., Tufft, M. R. A., Goodchild, H. L., \& Robbins, T. W. (2007). Differential effects of modafinil and methylphenidate on stop-signal reaction time task performance in the rat, and interactions with the dopamine receptor antagonist cis-flupenthixol. Psychopharmacology, 192, 193-206.

Eagle, D. M., \& Robbins, T. W. (2003). Inhibitory control in rats performing a stop-signal reaction-time task: Effects of lesions of the medial striatum and $d$-amphetamine. Behavioral Neuroscience, 117, 1302-1317.

Evenden, J. L. (1999). Varieties of impulsivity. Psychopharmacology, 146, 348-361.

Evenden, J. L., \& Ryan, C. N. (1996). The pharmacology of impulsive behaviour in rats: The 
effects of drugs on response choice with varying delays of reinforcement. Psychopharmacology, 128, 161-170.

Feola, T. W., de Wit, H., \& Richards, J. B. (2000). Effects of $d$-amphetamine and alcohol on a measure of behavioral inhibition in rats. Behavioral Neuroscience, 114, 838-848.

Green, L., \& Estle, S. J. (2003). Preference reversals with food and water reinforcers in rats. Journal of the Experimental Analysis of Behavior, 79, 233-242.

Green, L., Fisher, E. B., Perlow, S., \& Sherman, L. (1981). Preference reversal and self-control: Choice as a function of reward amount and delay. Behaviour Analysis Letters, 1, 43-51.

Green, L., Fristoe, N., \& Myerson, J. (2004). Temporal discounting and preference reversals in choice between delayed outcomes. Psychonomic Bulletin \& Review, 1, 383-389.

Green, L., \& Myerson, J. (2004) A discounting framework for choice with delayed and probabilistic rewards. Psychological Bulletin, 130, 769-792.

Green, L., Myerson, J., \& Macaux, E. W. (2005). Temporal discounting when the choice is between two delayed rewards. Journal of Experimental Psychology: Learning, Memory, and Cognition, 31, 1121-1133.

Hand, D. J., Fox, A. T., \& Reilly, M. P. (2009). Differential effects of $d$-amphetamine on impulsive choice in spontaneously hypertensive and Wistar-Kyoto rats. Behavioural Pharmacology, 20, 549-553.

Huskinson, S. L., Krebs, C. A., \& Anderson, K. G. (2011). Effects of acute and repeated administration of d-amphetamine on delay discounting in Lewis and Fischer 344 rats. Manuscript submitted for publication.

Kirby, K. N., \& Petry, N. M. (2004). Heroin and cocaine abusers have higher discount rates for delayed rewards than alcoholics or non-drug-using controls. Addiction, 99, 461-471. 
Kollins, S. H., MacDonald, E. K., \& Rush, C. R. (2001). Assessing the abuse potential of methylphenidate in nonhuman and human subjects: A review. Pharmacology Biochemistry and Behavior, 68, 611-627.

Logue, A.W. (1988). Research on self-control: an integrating framework. Behavioral and Brain Sciences, 11, 665-709.

Mazur, J. E. (1987). An adjusting procedure for studying delayed reinforcement. In M. L. Commons, J. E. Mazur, J. A. Nevin, \& H. Rachlin (Eds.), Qualitative analyses of behavior: The effect of delay and of intervening events on reinforcement value (Vol. 5, pp. 55-73), Hillsdale, NJ: Erlbaum.

Myerson, J., Green, L., \& Warusawitharana, M. (2001). Area under the curve as a measure of discounting. Journal of the Experimental Analysis of Behavior, 76, 235-243.

Odum, A. L., \& Baumann, A. A. L. (2010). Delay discounting: State and trait variable. In G. J. Madden \& W. K. Bickel (Eds.), Impulsivity (pp. 39-66), Washington, DC: American Psychological Association.

Perry, J. L., Stairs, D. J., \& Bardo, M. T. (2008). Impulsive choice and environmental enrichment: Effects of $d$-amphetamine and methylphenidate. Behavioural Brain Research, 193, 48-54.

Pietras, C. J., Cherek, D. R., Lane, S. D., Tcheremissine, O. V., \& Steinberg, J. L. (2003). Effects of methylphenidate on impulsive choice in adult humans. Psychopharmacology, 170, 390-398.

Pitts, R. C., \& Febbo, S. M. (2004). Quantitative analyses of methamphetamine's effects on selfcontrol choices: Implications for elucidating behavioral mechanisms of drug action. Behavioral Processes, 66, 213-233. 
Pitts, R. C., \& McKinney, P. A. (2005). Effects of methylphenidate and morphine on delaydiscounting functions obtained within sessions. Journal of the Experimental Analysis of Behavior, 83, 297-314.

Rachlin, H., \& Green, L. (1972). Commitment, choice, and self-control. Journal of the Experimental Analysis of Behavior, 17, 15-22.

Ramirez, J. M., \& Andreu, J. M. (2006). Aggression, and some related psychological constructs (anger, hostility, and impulsivity); some comments from a research project. Neuroscience and Biobehavioral Reviews, 30, 276-291.

Richards, J. B., Sabol, K. E., \& de Wit, H. (1999). Effects of methamphetamine on the adjusting amount procedure, a model of impulsive behavior in rats. Psychopharmacology, 146, 432-439.

Slezak, J. M., \& Anderson, K. G. (2009). Effects of variable training, signaled and unsignaled delays, and $d$-amphetamine on delay-discounting functions. Behavioural Pharmacology, 20, 424-436.

Vuchinich, R. E., \& Simpson, C. A. (1998). Hyperbolic temporal discounting in social drinkers and problem drinkers. Experimental Clinical Psychopharmacology, 6, 292-305.

Wade, T. R., de Wit, H., \& Richards, J. B. (2000). Effects of dopaminergic drugs on delayed reward as a measure of impulsive behavior in rats. Psychopharmacology, 150, 90-101.

Wilhelm, C. J., \& Mitchell, S. H. (2009). Strain differences in delay discounting using inbred rats. Genes Brain Behavior, 8, 426-434.

Winstanley, C. A., Dalley, J. W., Theobald, D. E., \& Robbins, T. W. (2003). Global 5-HT depletion attenuates the ability of amphetamine to decrease impulsive choice on a delay discounting task in rats. Psychopharmacology, 170, 320-331.

\section{John $\mathrm{H}$.}

Hagen 